\title{
Enteric pathogens induce tissue tolerance and prevent neuronal loss from subsequent infections
}

Tomasz Ahrends ${ }^{1}$, Begüm Aydin ${ }^{1}$, Fanny Matheis ${ }^{1}$, Cajsa Classon ${ }^{1,2}$, Gláucia C. Furtado ${ }^{3}$, Sérgio A. Lira ${ }^{3}$ and Daniel Mucida ${ }^{1}$

\footnotetext{
Affiliations:

${ }^{1}$ Laboratory of Mucosal Immunology, The Rockefeller University, New York, NY, USA.

2 Present address: Laboratory of Development and Homeostasis of the Nervous System, The Francis Crick Institute, London, UK.

${ }^{3}$ Precision Immunology Institute, Icahn School of Medicine at Mount Sinai, New York, NY, USA.

Correspondence should be addressed to T.A. (tahrends@rockefeller.edu) or to D.M. (mucida@rockefeller.edu)
} 


\begin{abstract}
The enteric nervous system (ENS) controls several intestinal functions including motility and nutrient handling, which can be disrupted by infection-induced neuropathies or neuronal cell death. We investigated possible tolerance mechanisms preventing neuronal loss and disruption in gut motility after pathogen exposure. We found that following enteric infections, muscularis macrophages (MMs) acquire a tissue-protective phenotype that prevents neuronal loss and dysmotility during subsequent challenge with unrelated pathogens. Bacteria-induced neuroprotection relied on activation of gut-projecting sympathetic neurons and signaling via $\beta_{2^{-}}$ adrenergic receptors $(\beta 2 \mathrm{AR})$ on $\mathrm{MMs}$. In contrast, helminth-mediated neuroprotection was dependent on T cells and systemic production of interleukin (IL)-4 and -13 by eosinophils, which induced arginase-expressing MMs that prevented neuronal loss from an unrelated infection located in a different intestinal region. Collectively, these data suggest that distinct enteric pathogens trigger a state of disease- or tissue tolerance that preserves ENS number and functionality.
\end{abstract}




\section{Introduction}

The gastrointestinal $(\mathrm{Gl})$ tract needs to simultaneously generate tolerance to harmless or beneficial dietary and microbial antigens and resistance to pathogen invasion. Additionally, disease tolerance to pathogen- and inflammation-induced tissue damage must operate in the intestine to maintain homeostasis (Medzhitov et al., 2012; Soares et al., 2014). This is particularly relevant for cells with reduced proliferative or regenerative capacity, such as intrinsic entericassociated neurons (iEANs), which are abundantly present in the gut and serve to modulate intestinal motility and secretory function (Balemans et al., 2017; Furness et al., 2013; O'Leary et al., 2019).

Previous studies in mice and rats, supported by clinical observations, indicate enteric infections can lead to neuronal loss, long-term dysmotility and neuropathies (Holschneider et al., 2011; Matheis et al., 2020; Ohman and Simren, 2010; White et al., 2018). Yet, whether a state of tolerance can be induced after exposure to pathogens, preventing cumulative neuronal loss and functional changes, is still not known.

We investigated possible tolerance mechanisms preventing neuronal loss and disruption in gut motility after pathogen exposure. We found that following helminth or bacterial infection and via distinct pathways MMs acquire tissue-protective qualities and prevent neuronal loss during subsequent challenge with an unrelated pathogen. Helminth-induced neuroprotection was dependent on $T$ cells and systemic production of interleukin (IL)- 4 and -13 by eosinophils and maintained long-term through the modulation of hematopoietic progenitors in the bone marrow. Conversely, bacteria-induced neuroprotection relied on $\beta_{2}$-adrenergic receptor signaling in MMs. Collectively, these data suggest that distinct enteric pathogens trigger a state of tolerance aimed to preserve the numbers and functionality of the ENS. 


\section{Results}

\section{Y. pseudotuberculosis infection induces iEAN protection during subsequent infection.}

To test whether primary infection can prevent neuronal loss after secondary challenge with an unrelated pathogen, we used an attenuated strain of Salmonella Typhimurium, spiB, which harbors a mutation in the type-III secretion system, impacting its intracellular replication (Tsolis et al., 1999). In contrast to wild-type Salmonella, spiB is not lethal and is cleared from wild-type C57BL/6 mice in 7-10 days, inducing a less severe form of Salmonellosis; nevertheless, spiB infection is sufficient to induce rapid iEAN loss and long-term dysmotility (Matheis et al., 2020). We thus first asked whether primary infection with an unrelated bacterium influences the tissue's response to a subsequent exposure to spiB. We used Yersinia pseudotuberculosis (Yp), which like Salmonella, is a foodborne Gram-negative bacterium that primarily localizes to the ileum (Fonseca et al., 2015), causing a transient infection undetectable in the feces by 21 days postinfection (dpi) (Figure 1A). Primary infection with Yp led to significant iEAN loss, however not as pronounced as after primary spiB infection. Additionally, as suggested by our recent studies (Matheis et al., 2020), spiB exposure following an earlier Yp infection did not result in further iEAN loss (Figure 1B). This was not a result of an improved resistance to spiB as mice previously infected with Yp displayed similar pathogen load and clearance pattern to naïve mice (Figure 1C).

Disease tolerance strategies alleviate the fitness costs by promoting resilience in the presence of an insult (Ayres, 2020). Because one of the main roles of the ENS in regard to fitness cost is the control of intestinal motility, we measured GI transit time as a functional readout for neuronal loss. Decreased iEAN numbers generally correlated with increased GI transit time; while spiB infection led to increased GI transit time, previous $Y p$ infection preserved gut motility after subsequent spiB challenge (Figure 1D). These results point to development of tissue-, or disease tolerance (Ayres, 2020; Martins et al., 2019) post-bacterial infection, which prevented neuronal loss to subsequent infection with a different pathogen. 
We have previously shown that MMs quickly acquire a tissue-protective phenotype and limit iEAN loss during primary spiB infection, a process that depends on $\beta_{2}$ adrenergic receptor $\left(\beta_{2}-A R\right)$ signaling on MMs (Gabanyi et al., 2016; Matheis et al., 2020). Our studies also pointed to Arginase 1-dependent polyamine production by MMs, which is induced upon infection in a $\beta_{2}$-ARdependent manner, mediating a basal neuroprotective effect during primary infections (Gabanyi et al., 2016; Matheis et al., 2020). To test whether Yp-induced tolerance relied on MMs, we used anti-CSF1R antibody, which selectively depletes MMs while preserving lamina propria macrophages (LpM) when administered at $50 \mathrm{mg} / \mathrm{kg}$ (Matheis et al., 2020; Muller et al., 2014). Administration of anti-CSF1R antibody to mice post Yp clearance led to a pronounced iEAN loss following challenge with spiB, suggesting the requirement for MMs to maintain $Y p$-induced neuronal protection (Figure 1E). Flow cytometric analysis of the ileum from mice after primary infection revealed that $Y p$ infection leads to the upregulation of Arg1 by MMs beyond a basal level (Figures 1F and 1G). To examine whether heightened Arg1 expression by MMs is required for Yp-induced neuroprotection, we used $\mathrm{LysM}^{\triangle \operatorname{Arg} 1}$ mice, in which $\operatorname{Arg} 1$ is conditionally deleted in myeloid cells. Yp-mediated neuroprotection was abolished in $\mathrm{LysM}^{\triangle A r g 1}$ following challenge with spiB infection (Figure $1 \mathrm{H}$ ). Moreover, this neuroprotective mechanism also required $b_{2}-A R$ expression by MMs, as LysM ${ }^{\triangle A d r b 2}$ mice previously infected with $Y p$ showed significant neuronal loss compared to wild-type littermate control mice upon spiB infection (Figure 1I). This effect correlated with decreased Arg1 expression by MMs and increased GI transit-time (Figure 1J and $1 \mathrm{~K})$. Consistently, while disease tolerance was impaired by both Arg 1 and $\beta_{2}$-AR targeting on MMs, spiB load was not affected by these strategies (Figures S1A and S1B). Taken together, these results indicate that primary bacterial infection results in a state of disease tolerance that mediates neuroprotection to subsequent infections, preserving intestinal motility. 
bioRxiv preprint doi: https://doi.org/10.1101/2021.04.09.439221; this version posted April 11, 2021. The copyright holder for this preprint (which was not certified by peer review) is the author/funder. All rights reserved. No reuse allowed without permission.

A

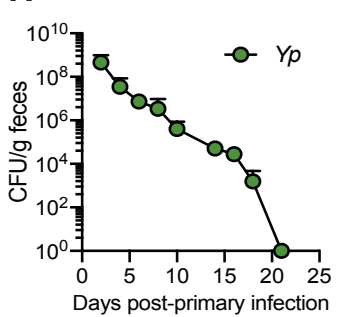

C

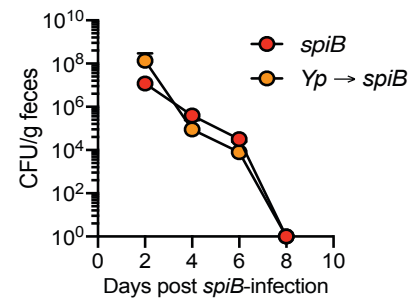

G

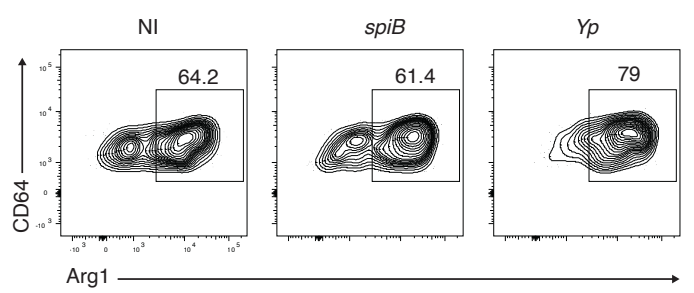

B

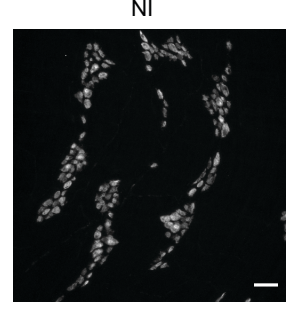

D

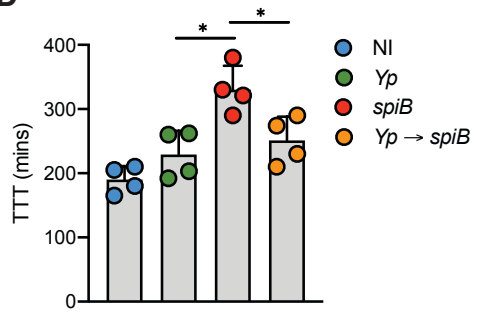

H

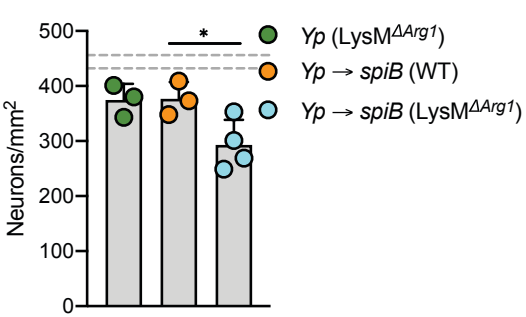

Yp

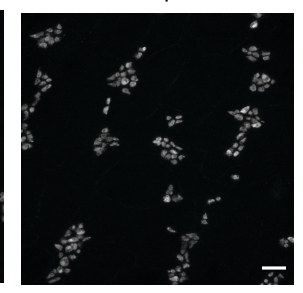

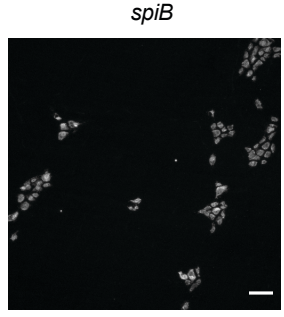

E

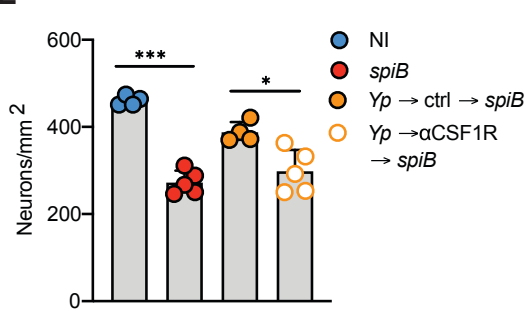

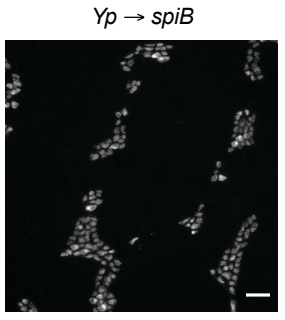

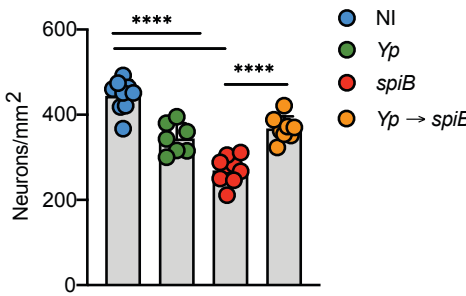

F

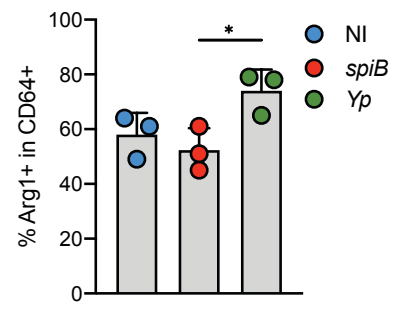

Figure 1. Y. pseudotuberculosis infection induces iEAN protection during subsequent infection.

(A-D) C57BL/6J mice were orally gavaged with PBS (non-infected, NI), $10^{9}$ colony-forming units (CFU) of Salmonella typhimurium spiB, $10^{8} \mathrm{CFU}$ of Y.pseudotuberculosis (Yp) only or $10^{8} \mathrm{CFU}$ of $Y p$ and 21 days later with $10^{9} \mathrm{CFU}$ of spiB. (A) Quantification of fecal Yp CFU. (B) Neuronal quantification in the ileum myenteric plexus assessed by IF staining of ANNA-1 on day 10 post-spiB infection. Left, representative images; Scale bars, $50 \mu \mathrm{m}$. (C) Quantification of fecal spiB CFU. (D) Total GI transit time measured at 12 days post infection (dpi). (E) C57BL/6J mice were orally gavaged with PBS, spiB or Yp. At 20 dpi Yp-infected mice were treated with anti-CSF1R or lgG isotype control, followed by oral gavage with spiB. lleum myenteric plexus neurons were quantified at 7 dpi. (F and $G$ ) Flow cytometry analysis of Arg1-expressing macrophages isolated from ileum muscularis at $14 \mathrm{dpi}$ with Yp or spiB. (H) Quantification of ileum myenteric plexus neurons of LysM ${ }^{\Delta A r g 1}$ mice and WT littermates orally gavaged with Yp only or followed by spiB infection 21 days later. (I-K) LysM ${ }^{\Delta A d r b 2}$ (Cre+) mice and WT (Cre-) littermates were orally gavaged with Yp and 21 days later with spiB. (I) Neuronal quantification of ileum myenteric plexus 7 days post-spiB infection. (J) Arg1 expression by macrophages in ileum muscularis at 7 dpi as determined by flow cytometry. (K) Total gastrointestinal (GI) transit time at $12 \mathrm{dpi}$. Dashed lines indicate the range of day $7 \mathrm{iEAN}$ numbers defined by mean $\pm \mathrm{SEM}$ of a large set of control $\mathrm{C} 57 \mathrm{BL} / 6 \mathrm{~J}$ mice. Data is from 2 pooled independent experiments (3-5 mice per condition, A to $C$ ) or one representative experiment (D-K). Error bars indicate SD, ${ }^{*} p \leq 0.05,{ }^{* *} p \leq 0.001,{ }^{* * * *} p \leq 0.0001$ (unpaired Student's t test or ANOVA with Tukey's post-hoc test). 
bioRxiv preprint doi: https://doi.org/10.1101/2021.04.09.439221; this version posted April 11, 2021. The copyright holder for this preprint (which was not certified by peer review) is the author/funder. All rights reserved. No reuse allowed without permission.
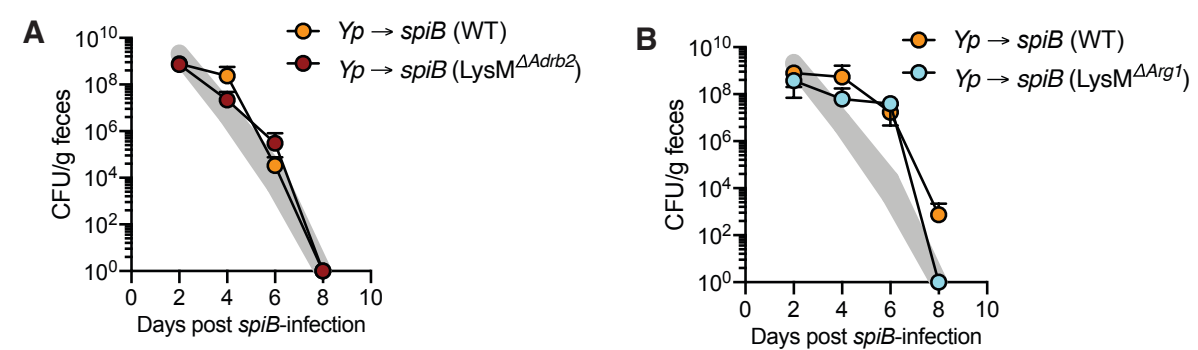

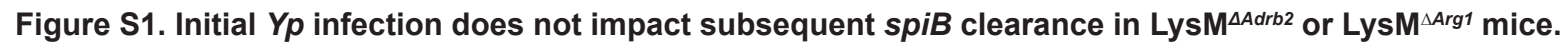

(A and B) LysM ${ }^{\triangle A d r b 2}$, LysM ${ }^{\triangle A r g 1}$ mice and WT littermates were orally gavaged with Y. pseudotuberculosis and 21 days later with Salmonella typhimurium spiB. Quantification of fecal CFU in $\operatorname{LysM}^{\triangle A d r b 2}(A)$ and LysM ${ }^{\Delta A r g 1}(B)$ mice. Shaded area indicates range of spiB CFU numbers defined by mean \pm SEM of a large set of control C57BL/6J mice. 


\section{Helminth infections induce long-term iEAN protection during subsequent infection.}

Next, we asked whether infection-induced neuroprotection could be induced by other pathogens. In particular, we aimed to determine whether helminths, which co-evolved with mammals and typically induce a very distinct immune response to bacterial pathogens, could prevent neuronal loss. We chose to infect mice with Strongyloides venezulenesis (Sv), a parasitic nematode that causes an acute infection and primarily localizes to the duodenum (Esterhazy et al., 2019; Silveira et al., 2002). Initial Sv infection was cleared by 12 dpi and, contrary to the bacterial pathogens we tested, did not lead to iEAN loss in the ileum or duodenum (Figures 2A, 2B, S2A, and (Matheis et al., 2020)). However, primary Sv duodenum infection completely prevented iEAN loss in the ileum following subsequent spiB challenge (Figures $2 \mathrm{~B}$ and $\mathrm{S} 2 \mathrm{~A}$ ). This distal protection by previous, proximal Sv infection was not associated with changes in spiB load and resulted in the maintenance of normal gut motility (Figures 2C and 2D). Additionally, while the proximal intestinehelminth Heligmosomoides polygyrus $(H p)$ was recently shown to impair anti-neurotropic virus immunity (Desai et al., 2021), distal protection from iEAN loss in the ileum was also observed in $H p$-infected and ivermectin-treated mice subsequently infected with spiB (Figure S2B). Moreover, initial Sv infection also led to neuroprotection after subsequent $Y p$ challenge (Figure S2C). Consistent with a disease tolerance phenotype, primary $S v$ or $H p$ infection did not affect subsequent clearance of spiB or $Y p$, respectively (Figures S2D and S2E).

Sv-induced tolerance depended on MMs, as evidenced by anti-CSF1R antibody-mediated depletion of MMs post Sv clearance, which abolished the neuroprotection upon spiB infection (Figure 2E). In line with our observations post primary Yp infection, following Sv challenge $\sim 80 \%$ of MMs expressed Arg1, compared to $\sim 60 \%$ observed after spiB infection (Figures $2 \mathrm{~F}$ and S2F). Of note, MMs in the duodenum expressed heightened Arg1 already at steady state compared to the ileum, and this was not further increased following spiB or Sv infection (Figure S2F). LysM ${ }^{\triangle A r g 1}$ mice previously infected with $S v$ showed significant neuronal loss following secondary spiB 
infection, in contrast to wild-type controls (Figure 2G). In opposition to $Y p$, Sv infection did not result in activation of gut-projecting sympathetic neurons (Figure $2 \mathrm{H}$ ). Conversely, in contrast to the phenotype observed post $Y p, S v$-mediated iEAN protection to subsequent spiB infection was maintained in LysM ${ }^{\triangle A d r b 2}$ mice. Mice lacking $\beta_{2}$-AR signaling in myeloid cells previously infected with $S v$ also sustained similar iEAN numbers, Arg1 expression by MMs, and GI transit time upon spiB infection as wild-type littermate controls (Figures 2I-2K). Moreover, initial infection with Sv did not affect spiB load and clearance in either LysM ${ }^{\triangle A d r b 2}$ and LysM ${ }^{\triangle A r g 1}$ mice (Figures S2G and $\mathrm{S} 2 \mathrm{H})$. These results indicate that while converging on neuroprotective MMs, $Y p$ - and $S v$-induced disease tolerance mechanisms are distinct.

We also examined how long Sv-mediated neuroprotection is maintained after the initial infection is cleared. Subsequent spiB challenge up to 12 weeks after primary Sv infection did not result in any iEAN loss. Challenge at 24 weeks post-infection did result in significant iEAN loss, however the enteric neuron numbers were still higher when compared to controls infected with spiB only. (Figure 2L). Long-term iEAN protection correlated with sustained high expression of Arg1 in MMs observed up to 12 weeks post-infection (Figure 2M). These results reveal a long-term neuroprotection in the ileum induced by a single duodenal helminth infection. 


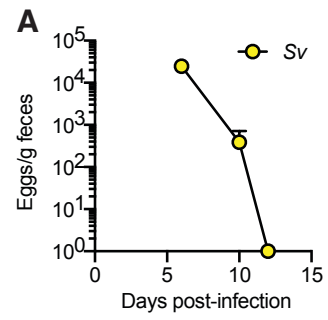

B

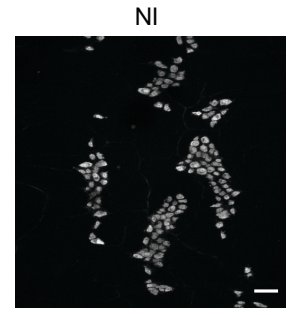

D

C

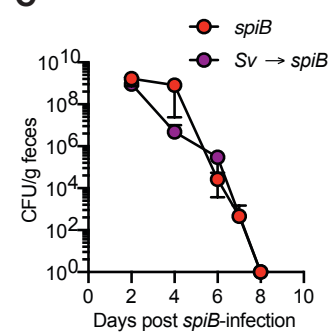

H
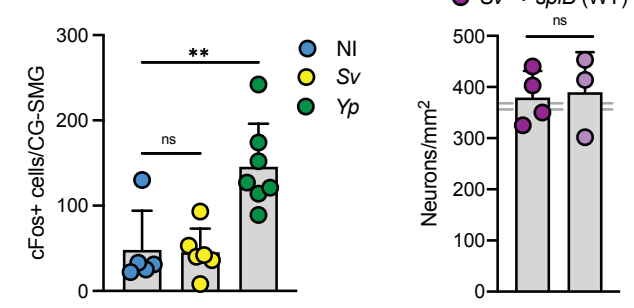

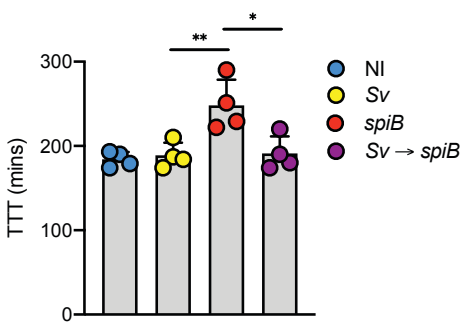

E
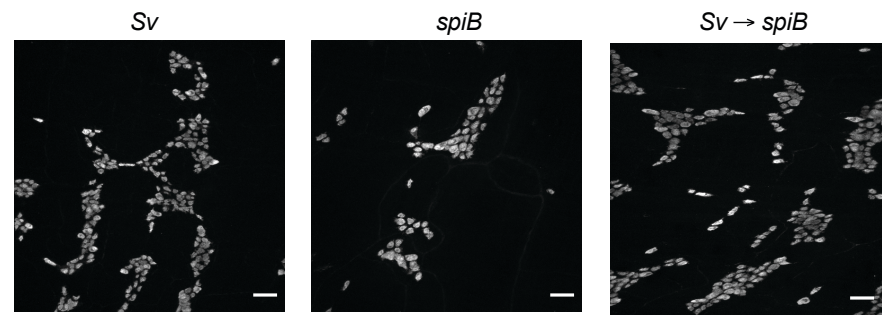

F
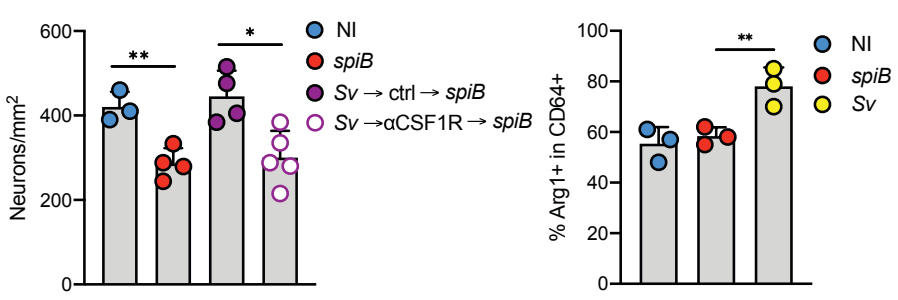

O $S_{v} \rightarrow$ spiB (WT) $\quad$ O $S v \rightarrow$ spiB (LysMMAdrb2)

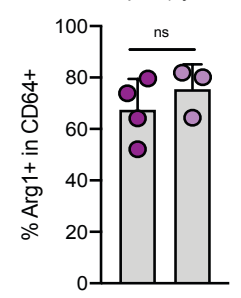

$\mathbf{L}$

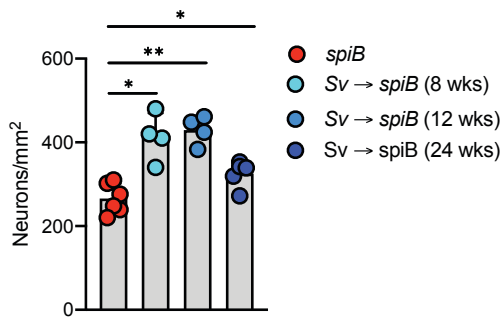

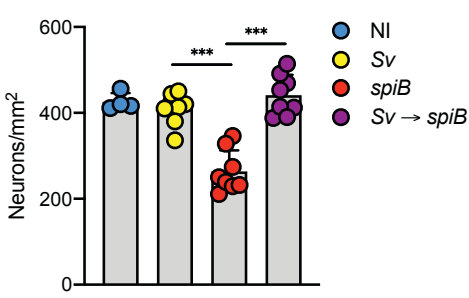

- $S v$ (LysM $\left.M^{\triangle A r g 1}\right)$

G $\quad 0 \quad s_{v} \rightarrow \operatorname{spiB}(\mathrm{WT})$

- $S v \rightarrow$ spiB $\left(\right.$ LysM $\left.^{\triangle A r g 1}\right)$

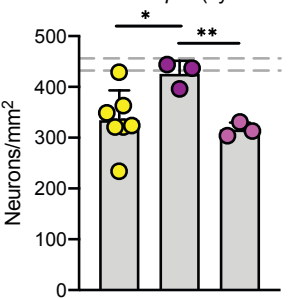

M

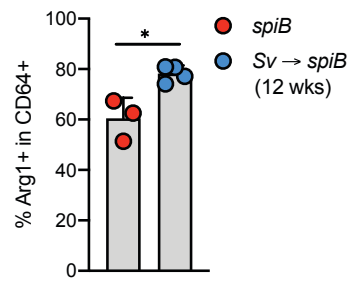

Figure 2. S. venezuelensis infection induces long-term iEAN protection during subsequent infection.

(A-D) C57BL/6J mice were s.c. injected with water and then orally gavaged with PBS (non-infected, NI) or infected with $10^{9}$ colony-forming units (CFU) of Salmonella Typhimurium spiB, $700 \mathrm{~S}$. venezuelensis (Sv) larvae only or $700 \mathrm{~Sv}$ larvae and 14 days later with $10^{9} \mathrm{CFU}$ of spiB. (A) Quantification of fecal Sv eggs. (B) Right, Neuronal quantification in the ileum myenteric plexus assessed by IF staining using ANNA-1 on day 8 post-spiB infection. Left, representative images; Scale bars, $50 \mu \mathrm{m}$. (C) Quantification of fecal spiB CFU. (D) Total GI transit time measured at $10 \mathrm{dpi}$. (E) C57BL/6J mice were orally gavaged with PBS or infected with spiB or Sv. At 14 dpi Sv-infected mice were treated with anti-CSF1R or lgG isotype control, followed by oral gavage with spiB. Ileum myenteric plexus neurons were quantified 7 days post-spiB infection. (F) Flow cytometry analysis of Arg1-expressing macrophages isolated from ileum muscularis at 14 dpi with Sv or spiB. (G) Neuronal quantification of ileum myenteric plexus of LysM ${ }^{\triangle A r g} 1(C r e+)$ mice and WT (Cre-) littermates orally gavaged with Sv only or followed by spiB infection 14 days later. $(\mathrm{H})$ Number of cFos+ neurons in CG-SMG 3 days after Yp infection, 7 days after Sv infection and in non-infected controls. (I-K) LysM ${ }^{\Delta A d r b 2}$ mice and WT littermates were orally gavaged with $S v$ and 14 days later with spiB. (I) Quantification of ileum myenteric plexus neurons 7 days post-spiB infection. (J) Arg1 expression by macrophages in ileum myenteric plexus at 7 dpi. (K) Total $\mathrm{Gl}$ transit time at 10 dpi. ( $\mathrm{L}$ and $\mathrm{M}$ ) C57BL/6J mice were orally gavaged with spiB only or Sv and 8, 12 or 24 weeks later with spiB. (L) lleum myenteric plexus neurons were quantified 10 days post-spiB infection. (M) Arg1 expression by macrophages in ileum myenteric plexus at 7 dpi as determined by flow cytometry. ( $G$ and I) Dashed lines indicate the range of day $7 \mathrm{iEAN}$ numbers defined by mean $\pm \mathrm{SEM}$ of all control C57BL/6J mice. Data is from 2 pooled independent experiments (3-5 mice per condition, A-C and H) or one representative experiment (D-G and I-M). Error bars indicate SD, ns - not significant, ${ }^{*} p \leq 0.05,{ }^{* *} p \leq 0.01,{ }^{* * *} p \leq 0.001$ (unpaired Student's t test or ANOVA with Tukey's post-hoc test). 
bioRxiv preprint doi: https://doi.org/10.1101/2021.04.09.439221; this version posted April 11, 2021. The copyright holder for this preprint (which was not certified by peer review) is the author/funder. All rights reserved. No reuse allowed without permission.

A

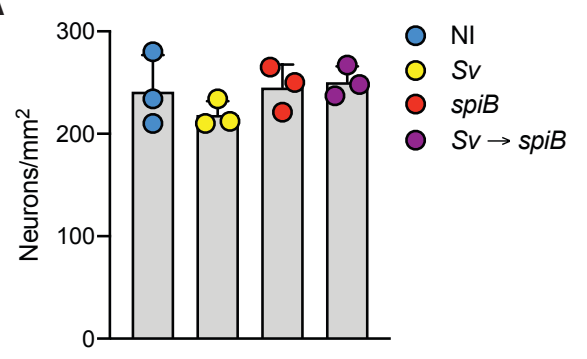

E

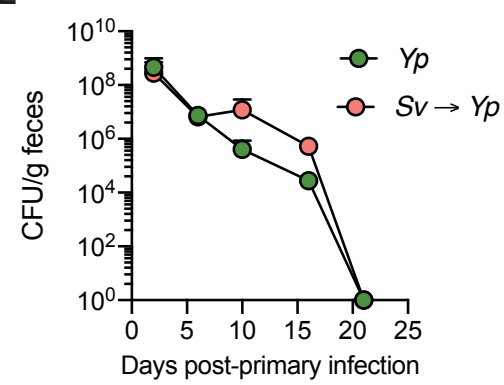

B

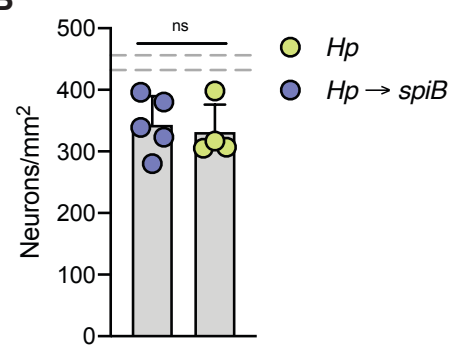

$\mathbf{F}$

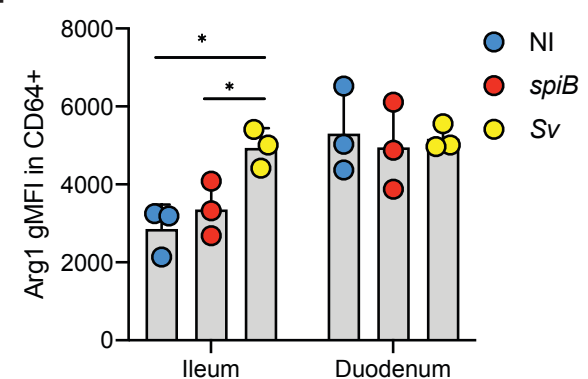

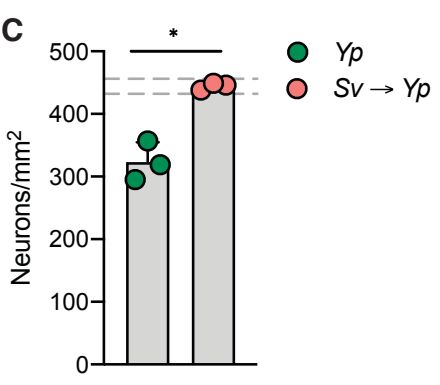

G
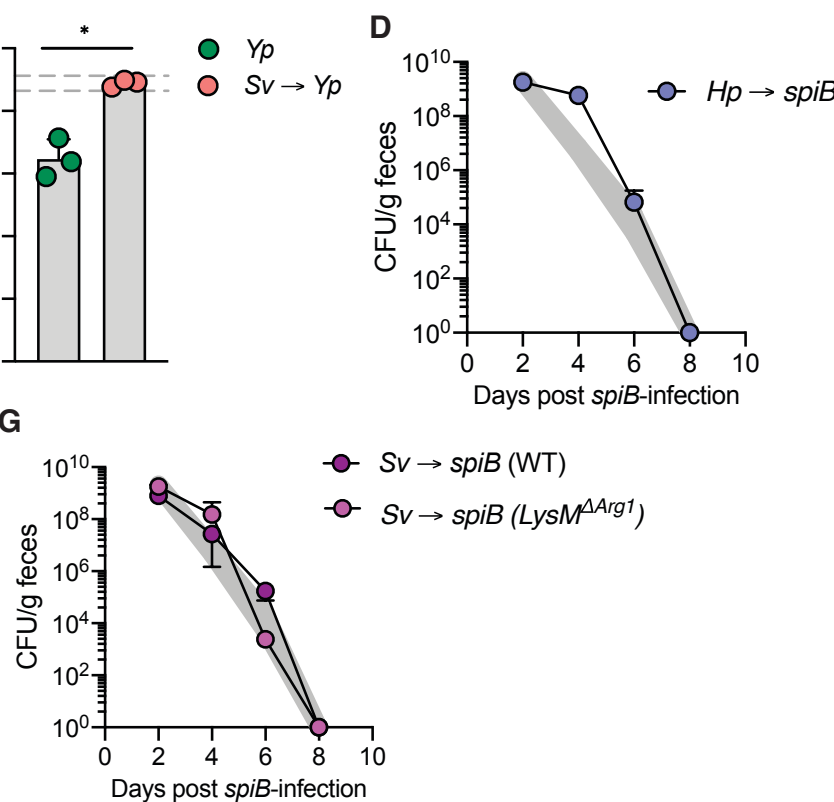

H

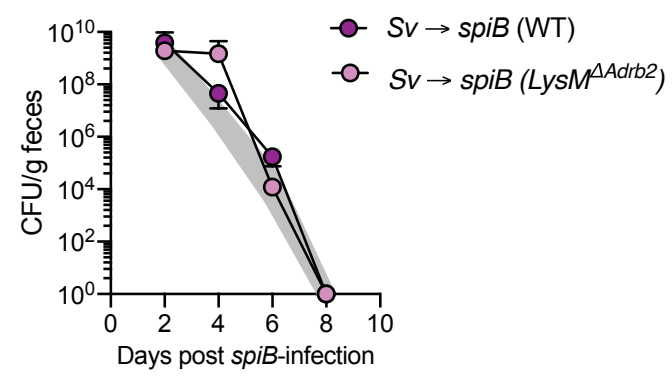

Figure S2. Helminth infections induce tissue tolerance.

(A) C57BL/6J mice were s.c. injected with water and then orally gavaged with PBS (non-infected, $\mathrm{NI}$ ), $10^{\circ}$ colony-forming units (CFU) of Salmonella spiB, or subcutaneously injected with $700 \mathrm{~S}$. venezuelensis (Sv) larvae only or $700 \mathrm{~Sv}$ larvae and 14 days later with $10^{\circ} \mathrm{CFU}$ of spiB. (A) Neuronal quantification in the duodenum myenteric plexus day 8 post-spiB infection. (B and C) C57BL/6J mice were orally gavaged with $200 \mathrm{H}$. polygyrus $(H p)$ larvae only or $H p$ larvae and 14 days later with spiB. (B) Quantification of ileum myenteric plexus neurons at 7 days post-spiB infection. (C) Quantification of fecal CFU. (D and E) C57BL/6J mice were orally gavaged with Yp or subcutaneously injected with Sv followed by $Y p 14$ days later. (D) Quantification of ileum myenteric plexus neurons at 21 days post-Yp infection. (E) Quantification of fecal CFU. (F) Frequencies of Arg1-expressing ileum and duodenum myenteric plexus macrophages in C57BL/6J mice orally gavaged with PBS (non-infected, NI), $10^{\circ}$ colony-forming units (CFU) of Salmonella spiB, or subcutaneously injected with $700 \mathrm{~S}$. venezuelensis (Sv) larvae. Flow cytometry analysis was performed at 14 days-post infection. ( $G$ and $H$ ) LysM ${ }^{\triangle A d r b 2}$, LysM ${ }^{\Delta A r g 1}$ mice and WT littermates were infected with Sv and 14 days later with spiB. Quantification of fecal CFU in LysM ${ }^{\triangle A r g 1}(G)$ and $\operatorname{LysM}^{\triangle A d r b 2}(\mathrm{H})$ mice. $(\mathrm{C}, \mathrm{G}$ and $\mathrm{H})$ Shaded area indicates range of spiB CFU numbers defined by mean \pm SEM of all control C57BL/6J mice. (B and D) Dashed lines indicate the range of day $7 \mathrm{iEAN}$ numbers defined by mean \pm SEM of a large set of control C57BL/6J mice. All data is representative of 2 independent experiments with 3-5 mice per condition. Error bars indicate SD, ns - not significant, * $p \leq 0.05$ (unpaired Student's t test or ANOVA with Tukey's post-hoc test). 


\section{Immune response to $S$. venezuelensis is associated with iEAN protection during subsequent infection.}

To identify a mechanism responsible for the long-term helminth-driven disease tolerance, we next further characterized the immune response to $S v$. Helminths are known to drive a robust type-2 immunity, with accumulation of innate immune cells, including tuft cells, eosinophils and group 2 innate lymphoid cells (ILC2), and later CD4 ${ }^{+}$T helper $2\left(T_{h} 2\right)$ cells (Maizels, 2020; O'Leary et al., 2019; Vivier et al., 2018). Coinfections with helminths may result in deviation of appropriate immune responses resulting in breakdown of barrier and increased susceptibility to unrelated pathogens (Desai et al., 2021; Marple et al., 2017). We observed an accumulation of Gata3 ${ }^{+}$CD4 ${ }^{+}$ " $\mathrm{T}_{\mathrm{h}} 2$ " cells in the duodenum, but not in the ileum LP following Sv infection, which was further increased when mice underwent subsequent spiB infection (Figures 3A and S3A). Additionally, we found a significant increase in the frequencies of mucosal mast cells and eosinophils in the LP and intraepithelial (IE) compartments of the ileum and duodenum of Sv-infected mice (Figures 3B, 3C, S3B, and S3C). At steady-state, however, the duodenum harbored higher numbers of eosinophils than the ileum, the former reaching almost $80 \%$ of total CD $45^{+}$cells found in the LP post $S v$-infection (Figures 3B and 3C). Of note, duodenal eosinophilia was maintained for up to 12 weeks post-infection and diminished at week 24 (Figure 3D), reflecting changes observed in MMs, GI transit time and neuroprotection post-Sv infection described above. Consistent with an overall heightened type-2 immunity, serum of $S v$-infected mice showed prolonged increase in interleukin (IL)-4 and IL-13 levels (Figures 3E and 3F).

We next examined whether $\mathrm{CD}^{+} \mathrm{T}$ cells were required for Sv-induced neuroprotection using a depleting anti-CD4 antibody following primary infection (Figure 3G). Depletion of CD4-expressing cells hindered type-2 immunity and the capacity to clear Sv infection. Therefore, we treated mice with the anti-helminthic drug, ivermectin, before infecting them with spiB (Figure $3 \mathrm{H}$ ). Mice treated with anti-CD4 antibody showed significant iEAN loss, which correlated with reduced eosinophilia 
and Arg1 expression by MMs (Figures 3I-3K). Tissue-resident memory $T\left(T_{R M}\right)$ cells mediate important in situ resistance functions and were also shown to provide cross-protection against different pathogens (Ariotti et al., 2014). We examined their role in Sv-induced neuroprotection by using mice with selective deficiency in the transcription factors Hobit and Blimp-1 (DKO mice), which were shown to be unable to generate $T_{R M}$ cells (Mackay et al., 2016). Compared to wildtype controls, DKO mice displayed no differences in iEAN numbers, frequencies of eosinophils and Arg1-expressing MMs after successive exposure to $S v$ and spiB, suggesting that $T_{\mathrm{RM}}$ cells do not play a significant role in this process (Figures S3D-S3F). These results indicate that CD4 ${ }^{+}$ T cells are required for $S v$-induced neuroprotection, and also point to their "accessory" or "helper" role in this process.

We then assessed whether the two main innate immune cell types that accumulate during helminth infections, mast cells and eosinophils, play a role in Sv-induced neuroprotection. Mast cells accumulation after Sv infection was predominantly observed in the duodenum epithelial compartment (Figure S3B). To target mast cells, we generated a novel CRISPR-based knock-in strain in which human diphtheria toxin receptor (hDTR) and the td tomato fluorescent protein are expressed under a promoter preferentially active in mast cells (Lilla et al., 2011), carboxypeptidase A3 (сpa3). Administration of diphtheria toxin (DT) after initial Sv infection led to a significant reduction in mast cell numbers (Figure S3G). Consistent with a role for mast cells in resistance to helminths, DT-treated mice showed a significant delay in Sv clearance (Figure S3H). Nevertheless, Sv-infected DT-treated $c p a 3^{\text {DTR-tdTomato }}$ and control mice showed similar iEAN numbers upon subsequent spiB challenge, suggesting that mast cells are dispensable for neuroprotection (Figure S3I). To address a possible role for eosinophils in this process, we first treated Sv-infected mice with neutralizing anti-IL-5 antibody followed by spiB infection. ILC2- and Th2-derived IL-5 drives eosinophil activation and accumulation (Maizels, 2020; O'Leary et al., 2019; Vivier et al., 2018); consistently, IL-5 blockade resulted in impaired eosinophilia (Figure 
bioRxiv preprint doi: https://doi.org/10.1101/2021.04.09.439221; this version posted April 11, 2021. The copyright holder for this preprint (which was not certified by peer review) is the author/funder. All rights reserved. No reuse allowed without permission.

S3J). Additionally, Sv-infected mice treated with anti-IL-5 displayed a significant iEAN loss and reduced Arg1 expression by MMs upon subsequent spiB exposure (Figures S3K and S3L). This data points to an important role for eosinophils in neuroprotection and disease tolerance post helminth infection. 
bioRxiv preprint doi: https://doi.org/10.1101/2021.04.09.439221; this version posted April 11, 2021. The copyright holder for this preprint (which was not certified by peer review) is the author/funder. All rights reserved. No reuse allowed without permission.

A
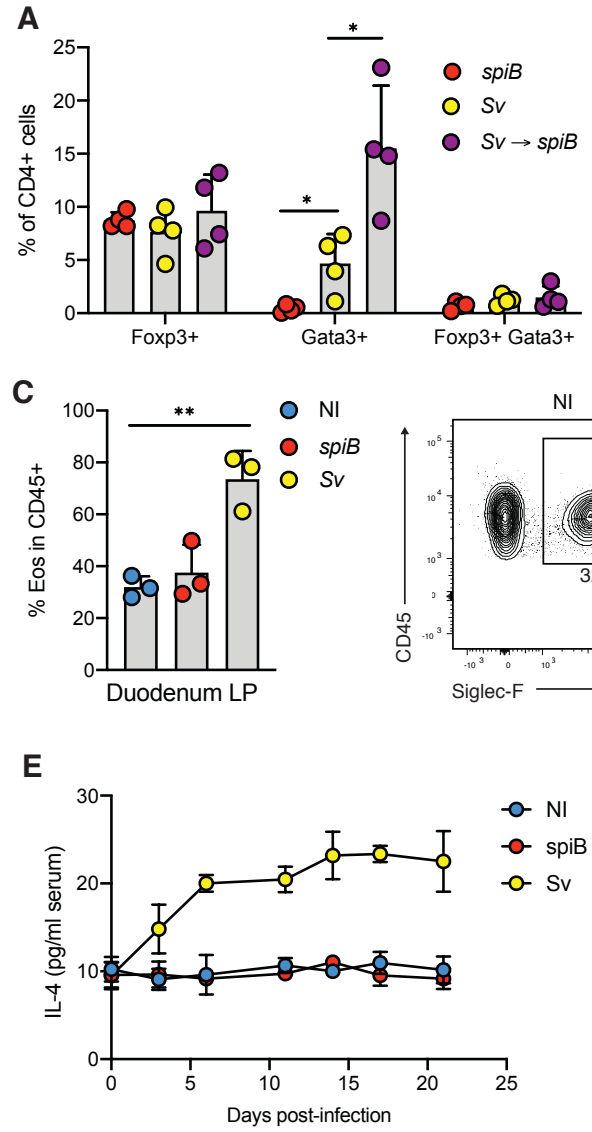

E

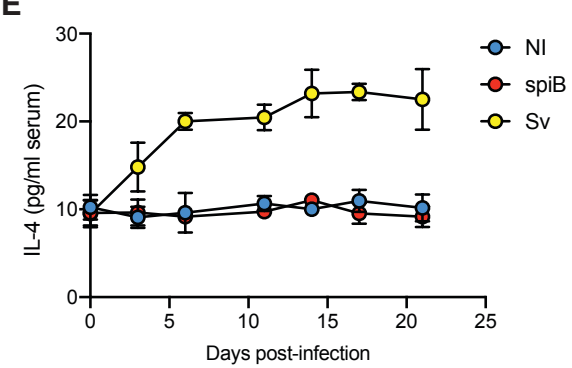

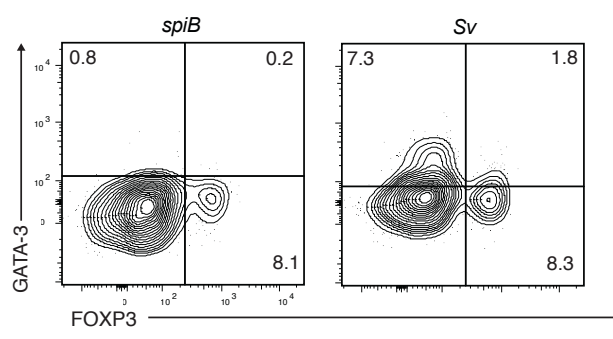

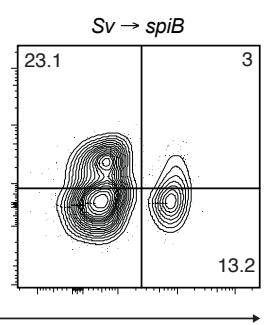

B

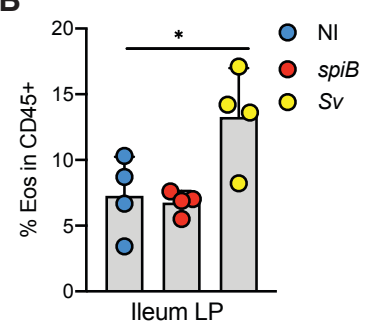

$\mathbf{F}$
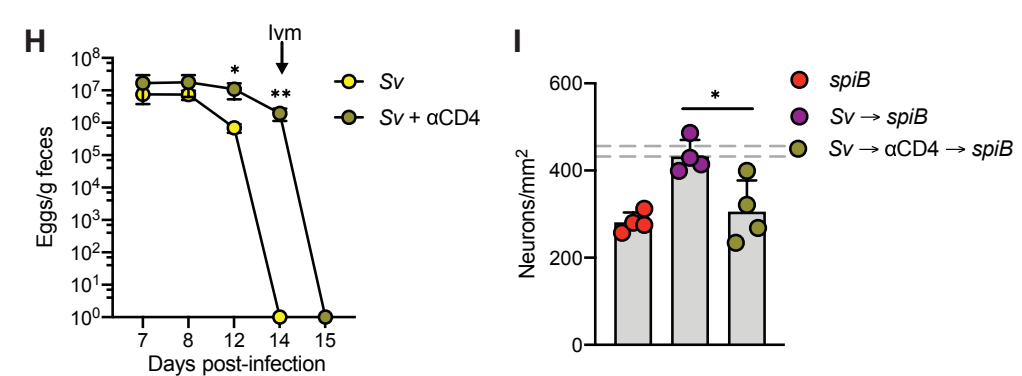

$\mathbf{J}$
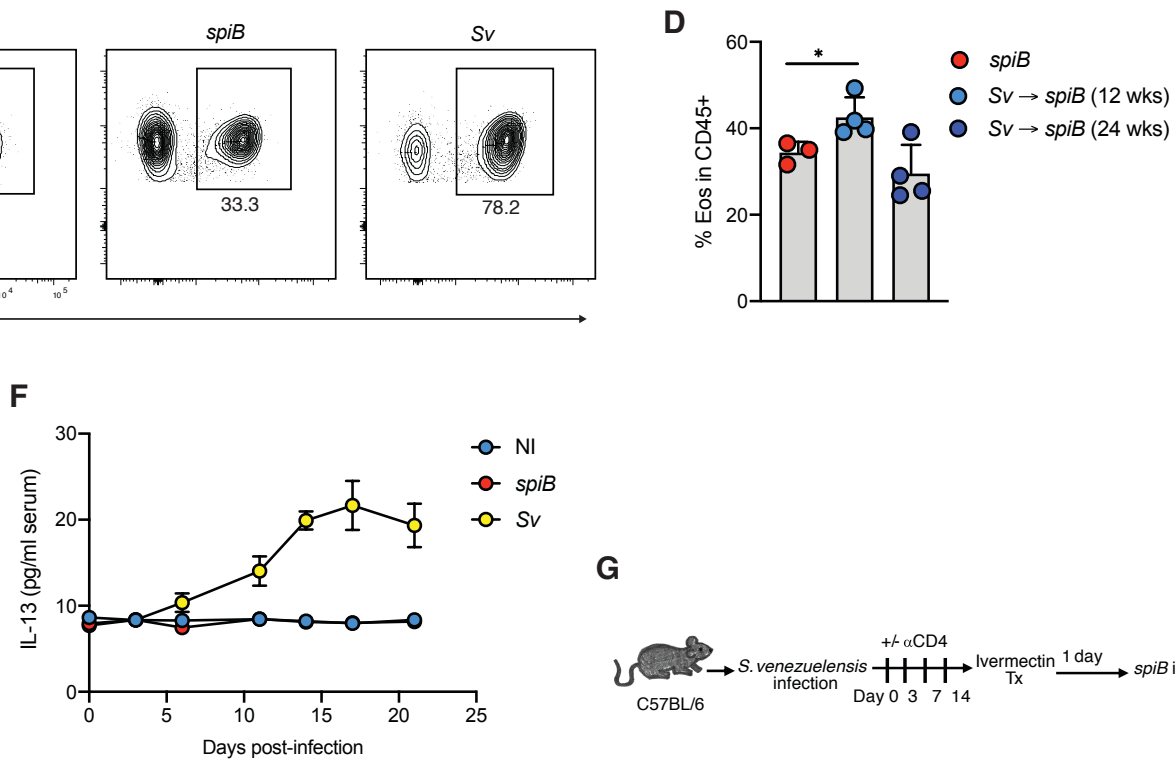

G

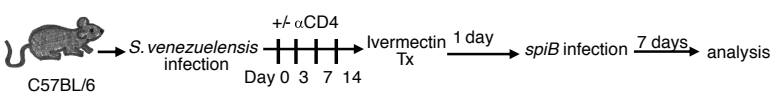

Figure 3. Immune response to $S$. venezuelensis is associated with iEAN protection during subsequent infection.

$(\mathrm{A}-\mathrm{F}) \mathrm{C}$ 78BL/6J mice were s.c. injected with water (non-infected, NI) or infected with spiB, Sv only or Sv and 14 days later with spiB. (A) Flow cytometric intranuclear analysis of CD4 ${ }^{+} \mathrm{T}$ cells in lamina propria (LP) expressing indicated transcription factors 2 days post-spiB infection. (B and C) Frequency of ileum (B) and duodenum (C) LP eosinophils at 14 dpi with Sv. (D) Frequency of duodenum LP eosinophils in mice infected with spiB only and Sv followed by spiB 12 or 24 weeks later and analyzed at 7 dpi. (E and F) Serum levels of IL-4 (E) and IL-13 (F) measured over time post-infection with spiB or Sv. (G) Experimental design for (H-K). (H-K) C57BL/6J mice were infected with spiB or Sv. On indicated days Sv-infected mice were treated with anti-CD4 antibody, followed by treatment with ivermectin and oral gavage with spiB. (H) Quantification of fecal Sv eggs. (I) Quantification of ileum myenteric plexus neurons 7 days post-spiB infection. Dashed lines indicate the range of day $7 \mathrm{iEAN}$ numbers defined by mean \pm SEM of a large set of control C57BL/6J mice. (J) Frequency of duodenum LP eosinophils at $7 \mathrm{dpi}$. (K) Arg1 expression by ileum myenteric plexus macrophages at 7 dpi. All data is representative of 2 independent experiments with 3-4 mice per condition. Error bars indicate SD, ${ }^{*} p \leq 0.05,{ }^{* *} p \leq 0.01,{ }^{* * *} p \leq 0.001$ (ANOVA with Tukey's post-hoc test). 
bioRxiv preprint doi: https://doi.org/10.1101/2021.04.09.439221; this version posted April 11, 2021. The copyright holder for this preprint (which

A

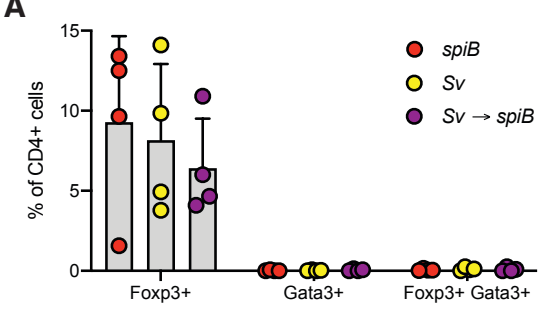

B

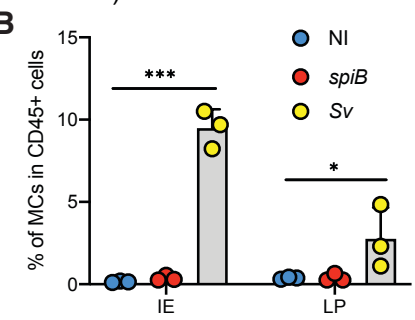

C

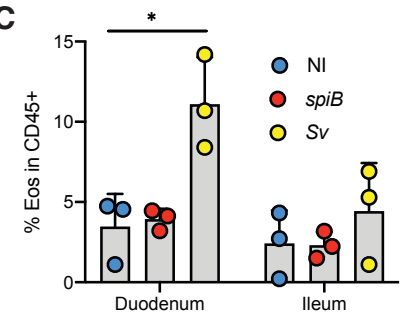

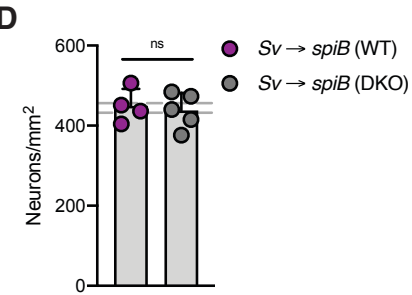

E

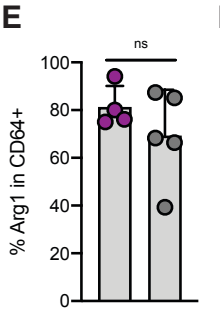

$\mathbf{F}$

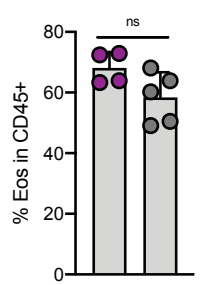

- $\mathrm{Sv} \rightarrow \mathrm{spiB}(\mathrm{WT})$

○ $\mathrm{Sv} \rightarrow \mathrm{spiB}(\mathrm{DKO})$

G

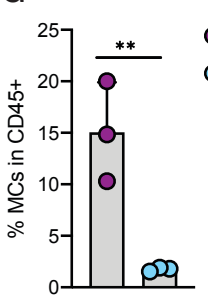

H

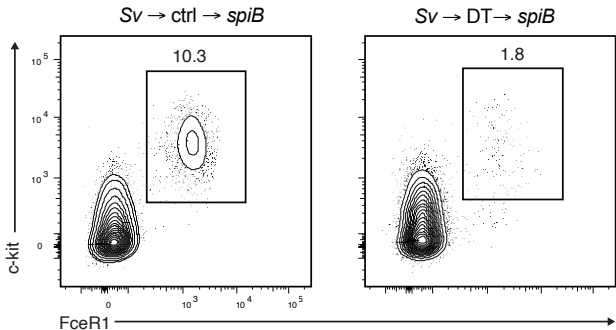

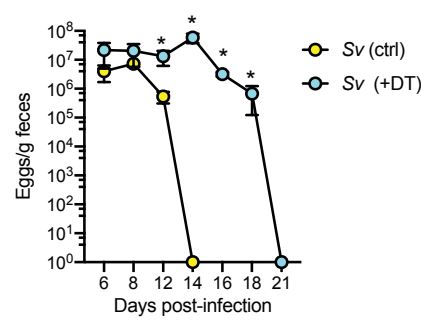

I

$\mathbf{J}$
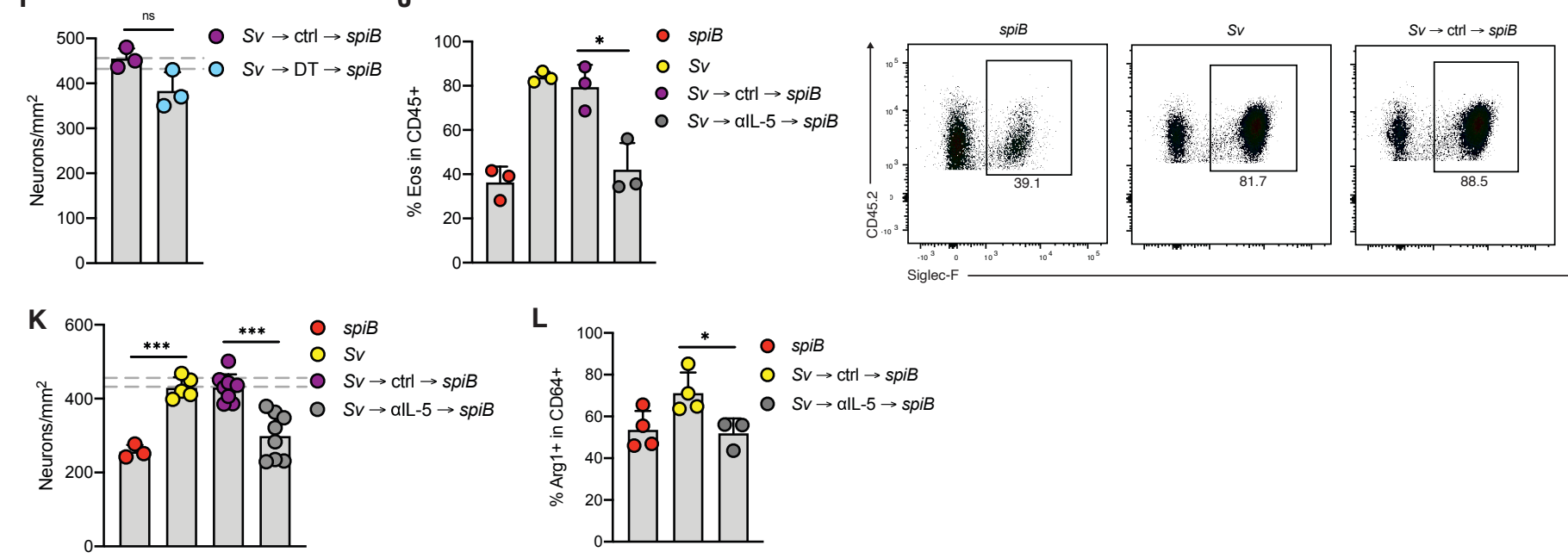

Figure S3. Helminth induced neuroprotection depends on IL-5.

(A-C) C57BL/6J mice were s.c. injected with water (non-infected, NI) or infected with spiB, Sv only or Sv and 14 days later with spiB. (A) Flow cytometry intranuclear analysis of $\mathrm{CD}^{+} \mathrm{T}$ cells in ileum lamina propria (LP) expressing indicated transcription factors 2 days post-spiB infection. (B) Frequencies of duodenum IE and LP mast cells at 14 dpi with Sv. (C) Frequencies of duodenum and ileum IE eosinophils at 14 dpi with Sv. (D-F)

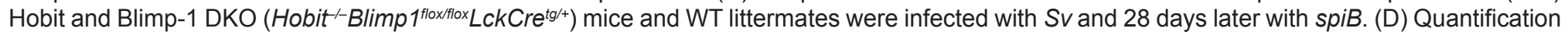
of ileum myenteric plexus neurons at $7 \mathrm{dpi}$ with spiB. (E) Arg1 expression by ileum myenteric plexus macrophages at 7 dpi. (F) Frequency of duodenum LP eosinophils at 7 dpi. (G-I) cpa3 ${ }^{\text {DTR-tdTomato }}$ mice and WT littermates were infected with Sv and treated with DT on day 0, 1, 4, 7, 10, 13, 16 and 18 post-infection. On day 21 post-Sv infection mice were orally gavaged with spiB. (G) Frequency of duodenum IE mast cells at 7 dpi with spiB. (H) Quantification of fecal Sv eggs. (I) Quantification of ileum myenteric plexus neurons at $7 \mathrm{dpi}$. (J-L) C57BL/6J mice were infected with spiB or Sv. Sv-infected mice were treated with anti-IL-5 antibody on day 0, 4, 8, 11 and 14 followed by oral gavage with spiB. Data is from one representative (A-D) or 2 pooled independent experiments with 3-5 mice per condition (G-J). Error bars indicate SD, ns - not significant, * $p \leq 0.05$ (unpaired Student's t test or ANOVA with Tukey's post-hoc test). 


\section{Pet store mice are resistant to neuronal loss upon spiB infection.}

We next turned to potential implications of infection-induced neuroprotection in free-living or nonlaboratory organisms, which are exposed to more pathogens and sustain a higher immune activation than laboratory animals (Abolins et al., 2017). We asked whether increased exposure to pathogens in mice maintained in a pet store resulted in altered iEAN numbers at steady state and loss upon bacterial infection. At steady state, pet store mice displayed lower iEAN numbers per $\mathrm{mm}^{2}$ when compared to naïve specific-pathogen-free (SPF) C57BL/6 mice (Figure 4A), resembling what we observed in SPF mice previously infected with Yp. However, we observed that pet store mice had significantly longer small intestines (Figure 4B). To account for these morphological differences, we analyzed the numbers of neurons found in each ganglion. Pet store mice had similar numbers of neurons per ganglion when compared to non-infected SPF controls (Figure 4C). Nevertheless, infection with spiB resulted in a decrease of both total number and neurons per ganglia in SPF, but not in pet store mice, although the latter also cleared spiB two days earlier than average SPF mice (Figures 4A, 4C and 4D). We noted a wide range in the frequency of eosinophils in the LP of pet store mice, probably reflecting their varied infection history (Figure 4E). Additionally, pet store mice showed overall elevated levels of serum IL-4 and IL-13, and Arg1 expression by MMs (Figures 4F and 4G). Finally, consistently with the increased frequency of eosinophils in the tissue, analysis of bone marrow of pet store mice revealed that both hematopoietic progenitors (Lineage $\left.{ }^{-} \mathrm{Sca} 1^{+} \mathrm{c}-\mathrm{Kit}^{+}, \mathrm{LSK}\right)$ cells and eosinophil progenitor (EoP) cells were enriched when compared to SPF controls (Figures $4 \mathrm{H}-4 \mathrm{~J}$ ). These results suggest that non-SPF mice, likely due to their infection history, acquire long-term protective mechanisms that regulate or prevent neuronal loss during infections occurring later in life. 
bioRxiv preprint doi: https://doi.org/10.1101/2021.04.09.439221; this version posted April 11, 2021. The copyright holder for this preprint (which was not certified by peer review) is the author/funder. All rights reserved. No reuse allowed without permission.

A

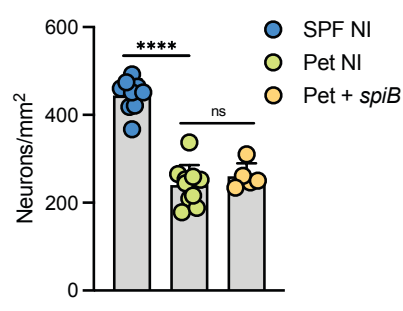

B

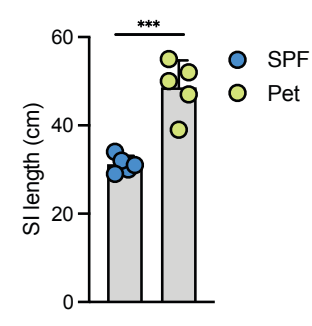

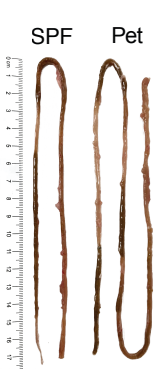

C

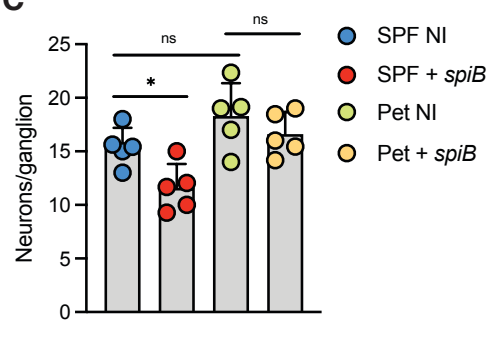

D

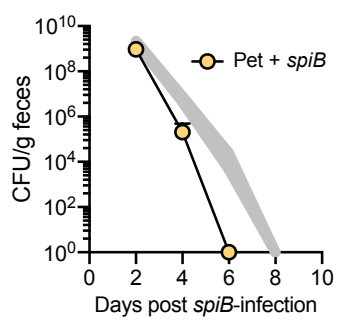

$\mathrm{E}$

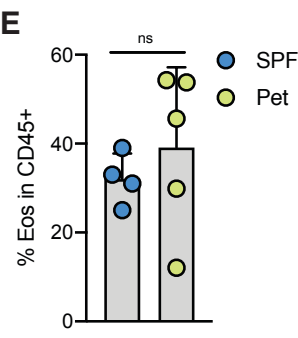

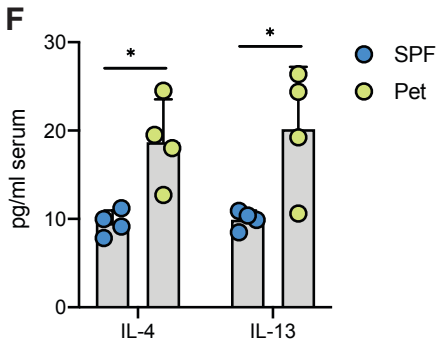

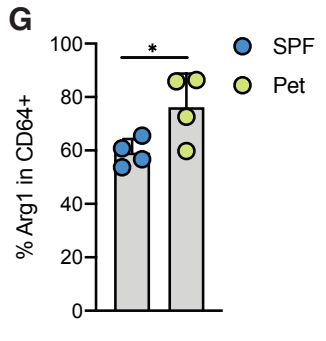

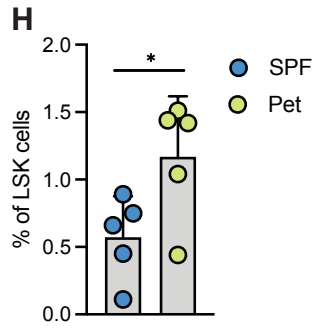

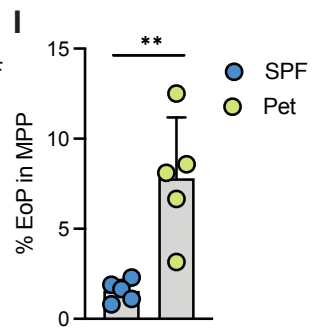

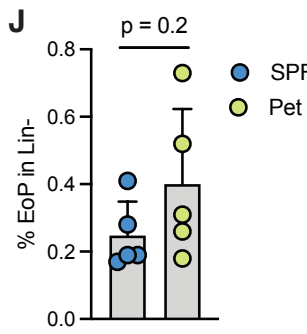

Figure 4. Pet store mice are resistant to neuronal loss upon spiB infection.

Pet store mice were analyzed at steady-state (A-C and E-J) or 7 days post spiB-infection (A, C and D). (A) Quantification of ileum myenteric plexus neurons. (B) Small intestine length. (C) Number of iEAN per ganglion within the ileum myenteric plexus. (D) Quantification of fecal spiB CFU. Shaded area indicates range of spiB CFU numbers defined by mean \pm SEM from a large set of control SPF mice. (E) Frequency of duodenum LP eosinophils. (F) Serum levels of IL-4 and IL-13. (G) Frequency of Arg1-expressing ileum myenteric plexus macrophages. $(\mathrm{H})$ Frequencies of indicated bone marrow progenitor cell subsets. Data is from one representative (B-J) or 2 pooled independent experiments with 3-5 mice per condition (A). Error bars indicate SD, ns - not significant, ${ }^{*} p \leq 0.05,{ }^{* *} p \leq 0.01,{ }^{* * *} p \leq 0.001,{ }^{* * * *} p \leq 0.0001$ (unpaired Student's $\mathrm{t}$ test or ANOVA with Tukey's post-hoc test). 


\section{IL-4- and IL-13-producing eosinophils mediate Sv-induced neuronal protection.}

To directly investigate the contribution of eosinophils to this process, we utilized a genetic mouse model in which hDTR is expressed through eosinophil peroxidase locus (iPHIL) (Jacobsen et al., 2014). Administration of DT to Sv-infected iPHIL mice significantly reduced the numbers of eosinophils in duodenum and ileum LP (Figures 5A and 5B). Depletion of eosinophils did not affect egg load and $S v$ clearance, but abolished $S v$-induced neuroprotection upon spiB infection (Figures 5C and 5D). This effect correlated with decreased expression of Arg1 by MMs and reduced GI motility (Figures 5E and 5F). In contrast, Yp-induced neuronal protection was not affected by depletion of eosinophils (Figures S4A and S4B).

Eosinophils are known to be major producers of IL-4 and IL-13, both of which can skew macrophages towards a tissue-protective phenotype (Liang et al., 2011; Wu et al., 2011). To evaluate the role of these cytokines produced by eosinophils in neuroprotection, we utilized $E p x^{\Delta / 4 ; / 113}$ mice, in which $/ / 4$ and $/ / 13$ genes are excised by eosinophil peroxidase promoter-driven Cre recombinase (Doyle et al., 2013). While Sv-induced eosinophilia was preserved in Epx $\Delta \underline{\Delta l 4 ; / 13}$ mice, subsequent spiB infection resulted in significant iEAN loss compared to wild-type control mice (Figures 5G and 5H). Consistently, absence of IL-4 and IL-13 in eosinophils resulted in decreased Arg1 expression by MMs and increased $\mathrm{Gl}$ transit time (Figures $5 \mathrm{I}$ and $5 \mathrm{~J}$ ). To investigate the exact localization of neuroprotective eosinophils, we performed a series of adoptive transfer experiments. Eosinophils derived from the ileum or duodenum from $S v$-infected mice were transferred to congenic hosts that were subsequently challenged with spiB (Figure $5 \mathrm{~K}$ ). Transfer of $6,5 \times 10^{5}$ duodenum eosinophils resulted in iEAN protection (Figures $5 \mathrm{~L}$ and $5 \mathrm{M}$ ). In contrast, equal numbers of ileum eosinophils failed to induce a neuroprotective effect upon spiB challenge; these effects correlated with increased expression of IL-4 by eosinophils derived from duodenum versus ileum of $S v$-infected mice (Figures $5 \mathrm{~N}$ and 50 ). These results suggest that 
secretion of IL-4 and/or IL-13 by activated duodenum eosinophils mediates distal neuroprotective effects by inducing Arg1 expression in MMs.

Finally, we asked whether long-term neuronal protection is dependent on constant replenishment of eosinophils and if newly recruited macrophages can acquire a tissue-protective phenotype. Following Sv infection, we treated mice with anti-CSF1R antibody and waited for new monocytes to be recruited to the tissue and differentiate into MMs (Gabanyi et al., 2016). Despite MM depletion post-Sv infection, subsequent spiB infection did not result in iEAN loss or reduced Arg1 expression by MMs when compared to isotype-treated animals, suggesting that long-term iEAN protection depends on eosinophils that can induce a protective phenotype in newly developed MMs (Figures S4C-S4E).

Eosinophils were shown to be able to survive up to 3 weeks within tissues, while $S v$-infection resulted in neuroprotection up to 24 weeks. Based on recent reports (Kaufmann et al., 2018; Mitroulis et al., 2018), we hypothesized that initial Sv infection modulates bone marrow hematopoietic stem and progenitor cells, skewing them towards eosinophilic lineage. We analyzed bone marrow of $S v$-infected mice and found that hematopoietic progenitors (LSK) cells, granulocyte macrophage progenitor (GMP) and eosinophil progenitor (EoP) cells were enriched up to 8 weeks post-infection (Figures S4F-S4J). These results indicate that Sv induces long-term restructuring of bone marrow precursors that can sustain an eosinophilic environment, dictating a protective phenotype in tissue macrophages. 
bioRxiv preprint doi: https://doi.org/10.1101/2021.04.09.439221; this version posted April 11, 2021. The copyright holder for this preprint (which was not certified by peer review) is the author/funder. All rights reserved. No reuse allowed without permission.

A

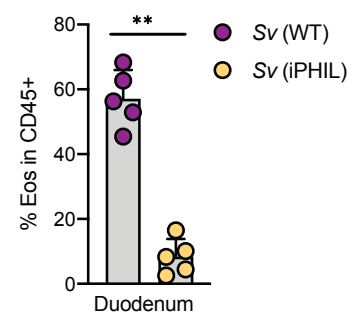

D

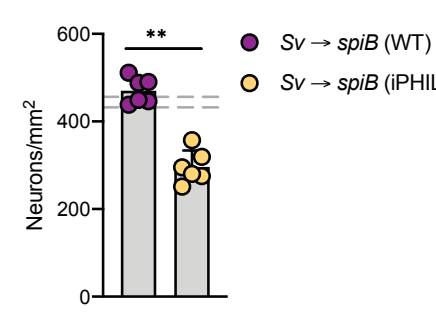

I

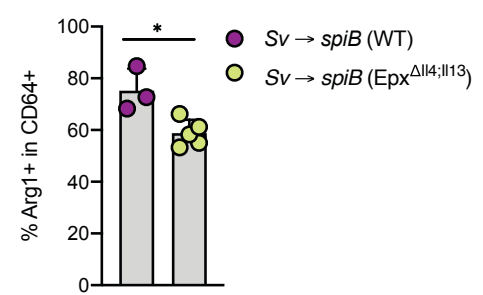

L

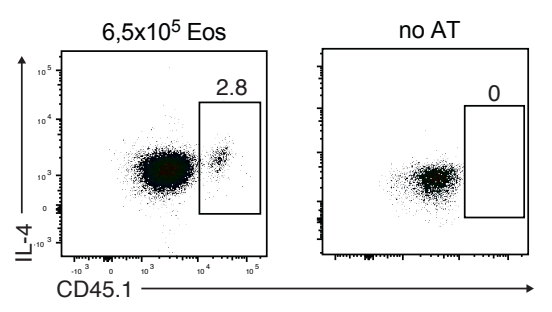

E

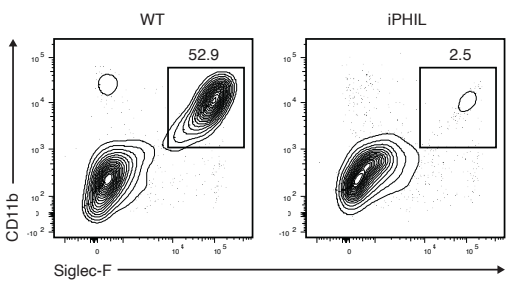

F

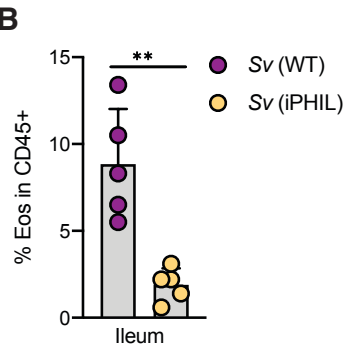

G

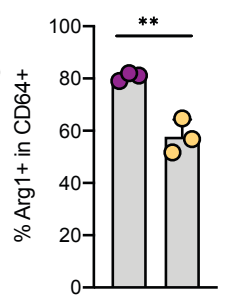

F

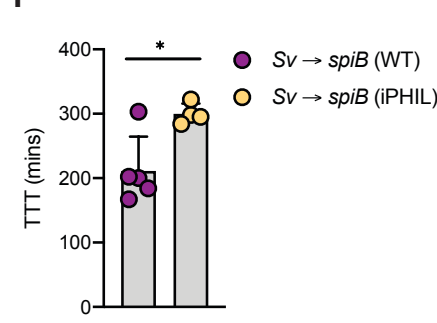

J
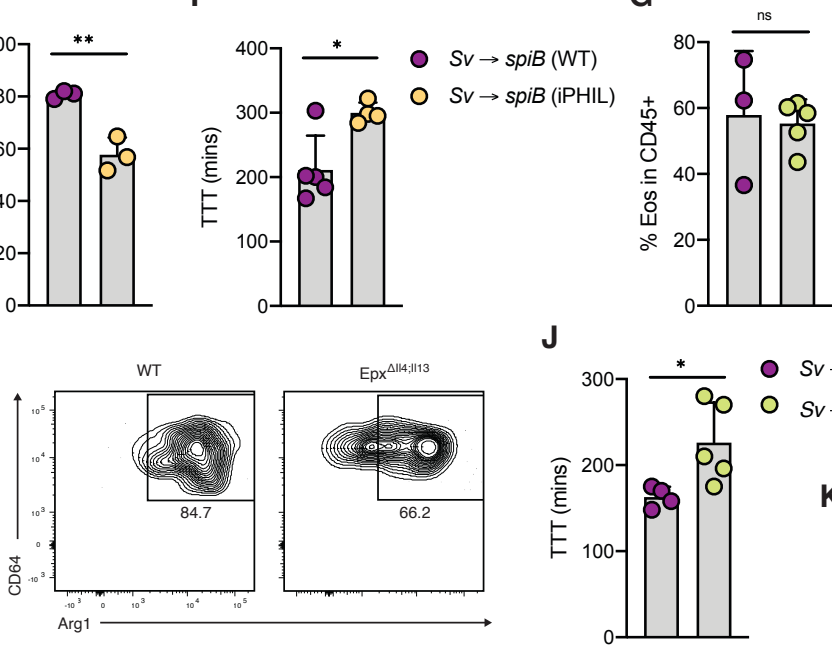

M

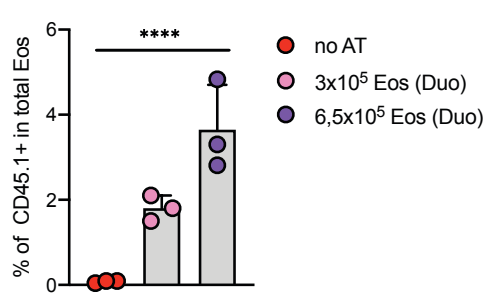

C

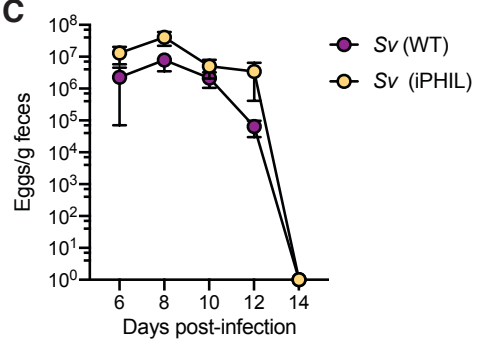

H

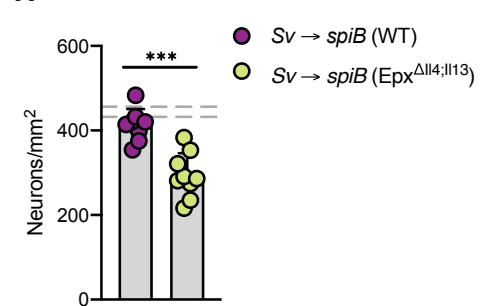

Sv $\rightarrow$ spiB (WT)

$S v \rightarrow \operatorname{spiB}\left(\mathrm{Epx}^{\Delta\|14 ;\| 113}\right)$

K
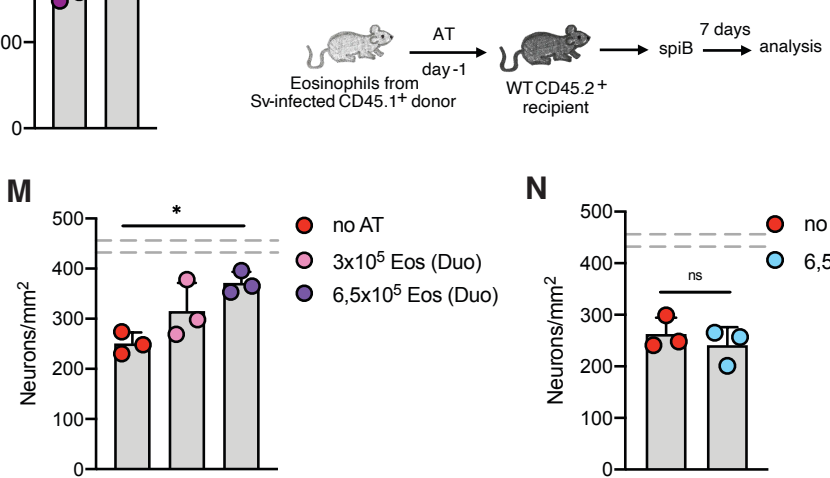

N

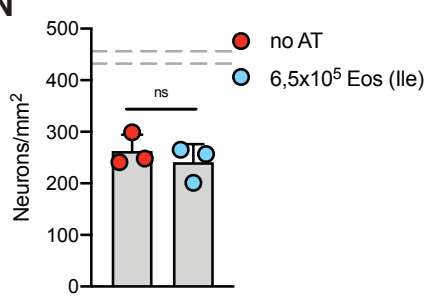

0

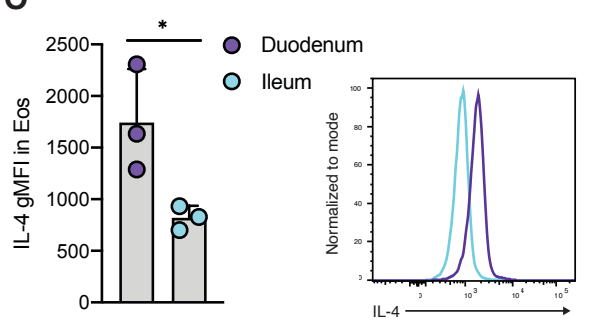

Figure 5. IL-4 and IL-13 producing eosinophils mediate Sv-induced neuronal protection.

(A-C) iPHIL mice and WT littermates were infected with $S v$ and treated with DT on day 0, 1, 4, 6 and 8 post-infection. Analysis was performed at 10 dpi. (A and B) Frequency of duodenum (A) and ileum (B) LP eosinophils. (C) Quantification of fecal Sv eggs. (D-F) iPHIL mice and WT littermates were infected with Sv and treated with DT on day $0,1,4,7,10,13,16$ and 18 post-infection. On day 14 post-Sv infection mice were orally gavaged with spiB. (D) Quantification of ileum myenteric plexus neurons at $7 \mathrm{dpi}$. (E) Arg1 expression by ileum myenteric plexus macrophages at 7 dpi. (F) Total GI transit time measured 10 days post-spiB infection. (G-J) Epx ${ }^{\Delta l 1 ; i l 13}$ mice or WT littermates were infected with Sv larvae and 14 days later with spiB. (G) Frequency of duodenum LP eosinophils at $7 \mathrm{dpi}$. (H) Quantification of ileum myenteric plexus neurons at $7 \mathrm{dpi}$. (I) Arg1 expression by ileum myenteric plexus macrophages at $7 \mathrm{dpi}$. (J) Total $\mathrm{Gl}$ transit time measured 10 days post-spiB infection. (K) Experimental design for (L-N). (L-N) C57BL/6J CD45.2 mice received indicated numbers of eosinophils isolated from duodenum or ileum LP of CD45.1 mice infected with Sv 7 days earlier. Recipient mice were subsequently infected with spiB. (L) Frequencies of donor CD45.1 $1^{+}$duodenum eosinophils found in LP of recipient mice at $7 \mathrm{dpi}$. (M) Quantification of ileum myenteric plexus neurons in mice receiving duodenum $(\mathrm{M})$ or ileum $(\mathrm{N})$ eosinophils 7 days post-spiB infection. (O) C57BL/6J mice were infected with Sv. Expression of IL-4 was measured in duodenum and ileum eosinophils 7 at dpi. Dashed lines indicate the range of day 7 iEAN numbers defined by mean \pm SEM of a large set of control C57BL/6J mice. Data is from one representative (A-G and I-O) or 2 pooled independent experiments with 3-5 mice per condition $(H)$. Error bars indicate SD, ns - not significant, ${ }^{*} p \leq 0.05,{ }^{* *} p \leq 0.01,{ }^{* * *} p \leq 0.0001$ (unpaired Student's t test or ANOVA with Tukey's post-hoc test). 
bioRxiv preprint doi: https://doi.org/10.1101/2021.04.09.439221; this version posted April 11, 2021. The copyright holder for this preprint (which was not certified by peer review) is the author/funder. All rights reserved. No reuse allowed without permission.

A

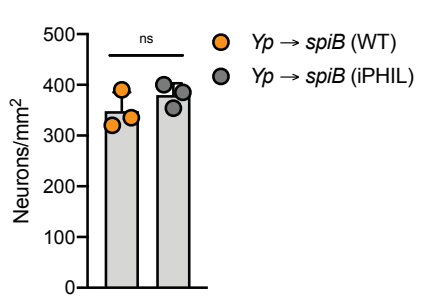

B

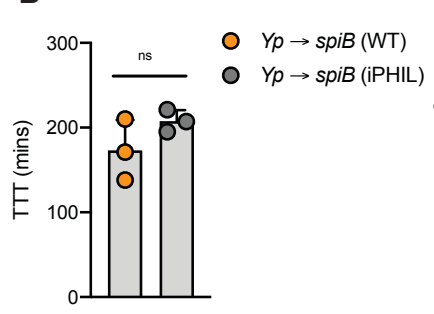

C

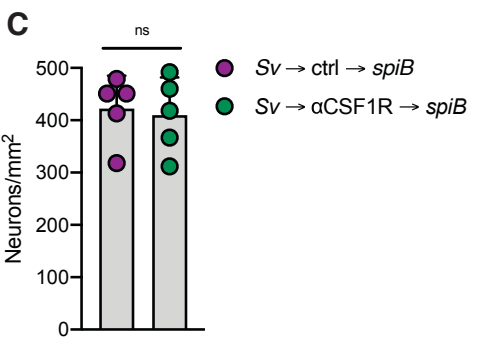

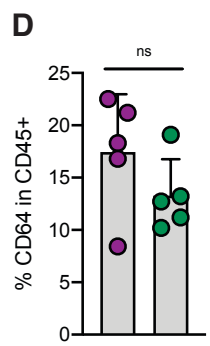

E

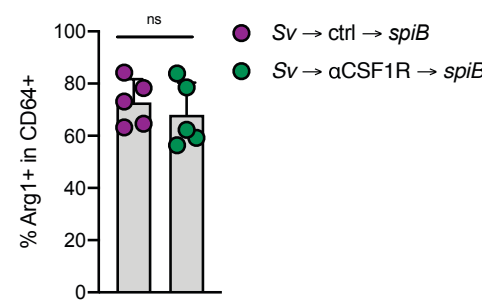

$\mathbf{F}$
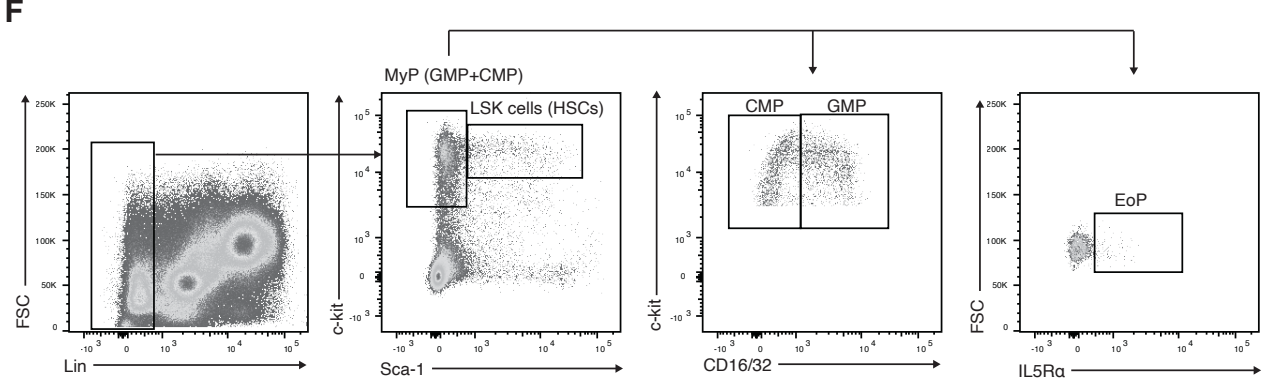

G

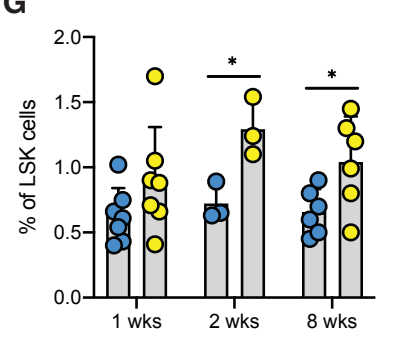

H

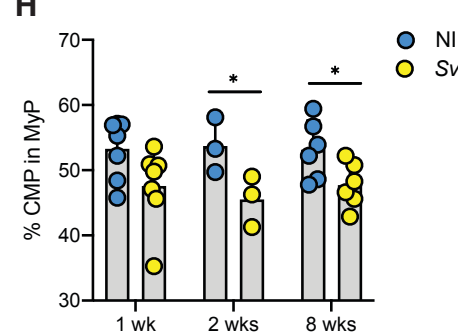

I

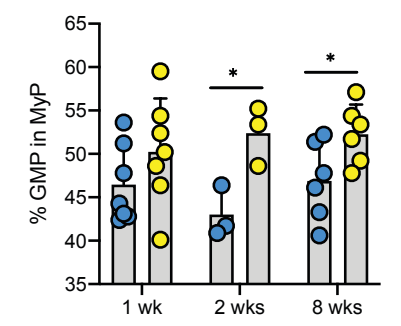

$J$

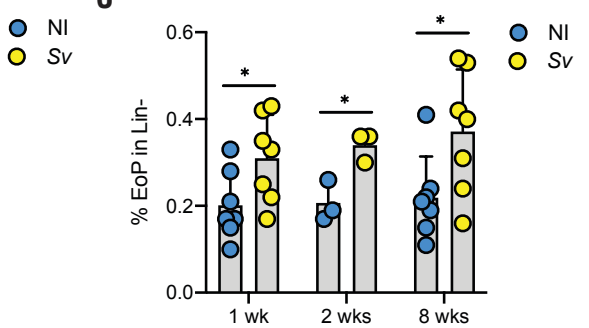

K

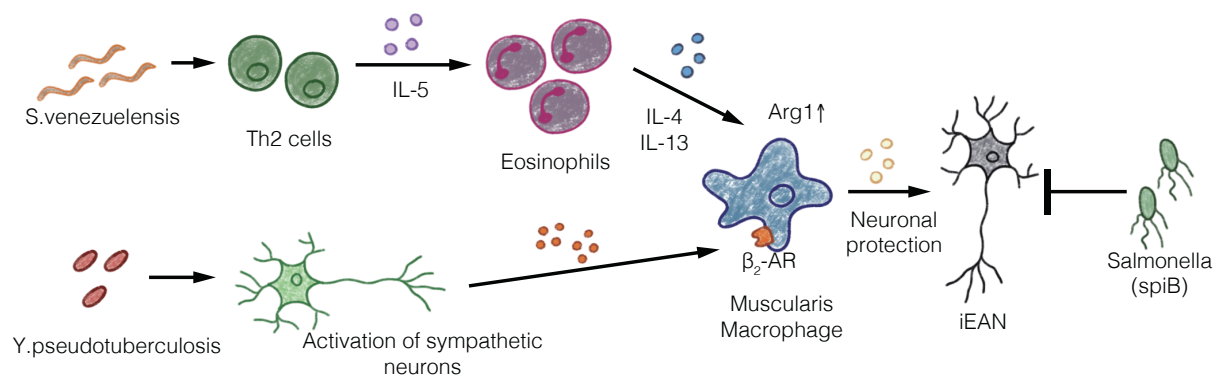

Figure S4. S. venezeluensis infection induces bone marrow hematopoietic progenitors.

(A and B) iPHIL mice and WT littermates were infected with $Y p$ and treated with DT on day 0, 1, 4, 7, 10,13, 16 and 18 post-infection. On day 21 post-Sv infection mice were orally gavaged with spiB. (A) Quantification of ileum myenteric plexus neurons at 7 dpi. (B) Total GI transit time measured 10 days post-spiB infection. (C-E) C57BL/6J mice were infected with Sv and at 14 dpi treated with anti-CSF1R or lgG isotype control antibodies, followed by oral gavage with spiB 14 days later. (C) lleum myenteric plexus neurons were quantified at 7 days post spiB-infection. (D) Frequency of ileum myenteric plexus macrophages at $7 \mathrm{dpi}$. (E) Frequency of Arg1-expressing ileum myenteric plexus macrophages at $7 \mathrm{dpi}$ with spiB. (F-J) Frequencies of indicated bone marrow progenitor cell subsets 1,2 and 8 weeks post-Sv infection. (K) Graphical representation of main findings. Bacteria-induced neuroprotection relied on $\beta 2$-adrenergic receptor signaling in MMs. Helminth-induced neuroprotection was dependent on T cells and systemic production of interleukin (IL)-4 and -13 by eosinophils, which regulate MMs located in a different intestinal region. These two pathways converged into Arginase-1 positive MMs that provide neuroprotection against subsequent infection.Data is from one representative (A-E) or 2 pooled independent experiments with 3-5 mice per condition (G-J). Error bars indicate SD, ns - not significant, * $p \leq 0.05$, ${ }^{* * * *} p \leq 0.0001$ (unpaired Student's t test or ANOVA with Tukey's post-hoc test). 


\section{Discussion}

Tissues can vary in their susceptibility to damage, regenerative capacity of their cells and in the impact caused by damage to host fitness; consequently, distinct disease tolerance mechanisms have been reported in different tissues and organs (Ayres, 2020; Martins et al., 2019; Medzhitov et al., 2012). The intestine is exposed to high amounts of microbes and potentially harmful substances, and consequently hosts highly regenerative cells like enterocytes, but also cells with low regenerative capacity such as neurons. While an increased regenerative capacity has been reported for enteric neurons, when compared to central nervous system (CNS) neurons (Furness, 2012), infection-induced neuronal loss can have a long-term impact in gut motility and host physiology (Balemans et al., 2017; Matheis et al., 2020; White et al., 2018).

Our findings raise the possibility that infections early in life determine the number of enteric neurons in adult life. Pet store mice had significantly longer small intestines, which has been shown to depend on elevated levels of systemic IL-4 and -13 and in models of chronic helminth infections (Schneider et al., 2018; Wong et al., 2007). Based on intestinal surface area and average neuronal densities, estimated total number of enteric neurons in the ileum is comparable between SPF and pet store mice, indicating that free-living animals might maintain the state of tissue tolerance due to constant exposure to different pathogens. Further studies are needed to define the impact of infections on iEAN during development, as well as the neurochemical code of neurons lost, and restored during episodes of microbe exposure throughout the life of animals (Furness, 2012).

The mechanistic studies described here indicate that the intestinal tissue co-opted pathways induced by distinct pathogens into a convergent tissue macrophage phenotype that mediates enteric neuronal protection, aiding host fitness via intestinal motility (Figure S4). Additionally, initial infections with enteric pathogens used here never affected subsequent bacterial burden and 
pathogen clearance. Thus, these observations fit the main defining characteristics of disease tolerance (Ayres, 2020; Martins et al., 2019; Medzhitov et al., 2012). The relatively short lifespan of eosinophils does not preclude their involvement in maintaining this state in the long term. This effect was most likely due to epigenetic modulation of myelopoiesis progenitors skewing them towards eosinophilic lineage. Of note, similar mechanisms have until now been mostly described in macrophages and their involvement in disease resistance (Kaufmann et al., 2018; Mitroulis et al., 2018). We hypothesize that certain infections can 'educate' HSCs in bone marrow resulting in increased eosinophilic output. As suggested by our comparison between duodenum versus ileal eosinophils, the local microenvironment primed by initial infection might then lead to additional eosinophil activation and secretion of cytokines that modulate tissue macrophages. While the impact of disease tolerance mechanisms for host fitness have been demonstrated against a wide range of pathogens, our observations indicate that disease tolerance induced upon infection can have a long-term effect that benefit the host upon subsequent encounter with unrelated pathogens.

\section{Author Contributions}

T.A. initiated, designed, performed the research and wrote the manuscript. B. A., F.M. and C.C. designed and performed experiments. G.F., S.L. generated the cpa3 ${ }^{\text {DTR-tdTomato }}$ mice. D.M. conceived, initiated, designed, supervised the research, and wrote the manuscript. All authors revised and edited the manuscript and figures.

\section{Acknowledgements}

We thank all Mucida Lab members and Rockefeller University employees for their continuous assistance; A. Rogoz and S. Gonzalez for the maintenance of mice and RU Bio-imaging Research Center for assistance with image acquisition analysis. We thank E. Jacobsen for 
providing iPHIL and Epx ${ }^{\mathrm{tm} 1.1(\mathrm{cre}) \text { Jlee }}$ mouse strains, M. Merad for the anti-CSF1R hybridoma, V.

Lenon for anti-Anna-1 antibody, S. Galli and K. Matsushita for the Sv larvae, I. Brodsky for Yp,

J. Lafaille for $H$. polygyrus larvae. We also thank Victora and Lafaille labs for fruitful discussions.

This work was supported by NIH Transformative R01DK116646 and R01DK126407, Kenneth

Rainin Foundation, Food Allergy FARE/FASI Consortium, NIH R01DK126407 (D.M.). T.A. is a

Human Frontier of Science Program postdoctoral fellow. B.A. is a Simons Foundation junior

fellow.

\section{References}

Abolins, S., King, E.C., Lazarou, L., Weldon, L., Hughes, L., Drescher, P., Raynes, J.G., Hafalla, J.C.R., Viney, M.E., and Riley, E.M. (2017). The comparative immunology of wild and laboratory mice, Mus musculus domesticus. Nature communications 8, 14811.

Ariotti, S., Hogenbirk, M.A., Dijkgraaf, F.E., Visser, L.L., Hoekstra, M.E., Song, J.Y., Jacobs, H., Haanen, J.B., and Schumacher, T.N. (2014). T cell memory. Skin-resident memory CD8(+) T cells trigger a state of tissue-wide pathogen alert. Science 346, 101-105.

Ayres, J.S. (2020). The Biology of Physiological Health. Cell 181, 250-269.

Balemans, D., Mondelaers, S.U., Cibert-Goton, V., Stakenborg, N., Aguilera-Lizarraga, J., Dooley, J., Liston, A., Bulmer, D.C., Vanden Berghe, P., Boeckxstaens, G.E., et al. (2017). Evidence for long-term sensitization of the bowel in patients with post-infectious-IBS. Scientific reports 7,13606 .

Bilate, A.M., Bousbaine, D., Mesin, L., Agudelo, M., Leube, J., Kratzert, A., Dougan, S.K., Victora, G.D., and Ploegh, H.L. (2016). Tissue-specific emergence of regulatory and intraepithelial T cells from a clonal T cell precursor. Sci Immunol 1, eaaf7471.

Desai, P., Janova, H., White, J.P., Reynoso, G.V., Hickman, H.D., Baldridge, M.T., Urban, J.F., Jr., Stappenbeck, T.S., Thackray, L.B., and Diamond, M.S. (2021). Enteric helminth coinfection enhances host susceptibility to neurotropic flaviviruses via a tuft cell-IL-4 receptor signaling axis. Cell.

Doyle, A.D., Jacobsen, E.A., Ochkur, S.I., Willetts, L., Shim, K., Neely, J., Kloeber, J., Lesuer, W.E., Pero, R.S., Lacy, P., et al. (2013). Homologous recombination into the eosinophil peroxidase locus generates a strain of mice expressing Cre recombinase exclusively in eosinophils. Journal of leukocyte biology 94, 17-24.

Esterhazy, D., Canesso, M.C.C., Mesin, L., Muller, P.A., de Castro, T.B.R., Lockhart, A., ElJalby, M., Faria, A.M.C., and Mucida, D. (2019). Compartmentalized gut lymph node drainage dictates adaptive immune responses. Nature 569, 126-130. 
Fonseca, D.M., Hand, T.W., Han, S.J., Gerner, M.Y., Glatman Zaretsky, A., Byrd, A.L., Harrison, O.J., Ortiz, A.M., Quinones, M., Trinchieri, G., et al. (2015). Microbiota-Dependent Sequelae of Acute Infection Compromise Tissue-Specific Immunity. Cell 163, 354-366.

Furness, J.B. (2012). The enteric nervous system and neurogastroenterology. Nature reviews Gastroenterology \& hepatology 9, 286-294.

Furness, J.B., Rivera, L.R., Cho, H.J., Bravo, D.M., and Callaghan, B. (2013). The gut as a sensory organ. Nature reviews Gastroenterology \& hepatology 10, 729-740.

Gabanyi, I., Muller, P.A., Feighery, L., Oliveira, T.Y., Costa-Pinto, F.A., and Mucida, D. (2016). Neuro-immune Interactions Drive Tissue Programming in Intestinal Macrophages. Cell 164, 378-391.

Geslewitz, W.E., Percopo, C.M., and Rosenberg, H.F. (2018). FACS isolation of live mouse eosinophils at high purity via a protocol that does not target Siglec F. Journal of immunological methods $454,27-31$.

Holschneider, D.P., Bradesi, S., and Mayer, E.A. (2011). The role of experimental models in developing new treatments for irritable bowel syndrome. Expert review of gastroenterology \& hepatology 5, 43-57.

Jacobsen, E.A., Lesuer, W.E., Willetts, L., Zellner, K.R., Mazzolini, K., Antonios, N., Beck, B., Protheroe, C., Ochkur, S.I., Colbert, D., et al. (2014). Eosinophil activities modulate the immune/inflammatory character of allergic respiratory responses in mice. Allergy 69, 315-327.

Kaufmann, E., Sanz, J., Dunn, J.L., Khan, N., Mendonca, L.E., Pacis, A., Tzelepis, F., Pernet, E., Dumaine, A., Grenier, J.C., et al. (2018). BCG Educates Hematopoietic Stem Cells to Generate Protective Innate Immunity against Tuberculosis. Cell 172, 176-190 e119.

Liang, H.E., Reinhardt, R.L., Bando, J.K., Sullivan, B.M., Ho, I.C., and Locksley, R.M. (2011). Divergent expression patterns of IL-4 and IL-13 define unique functions in allergic immunity. Nature immunology 13, 58-66.

Lilla, J.N., Chen, C.C., Mukai, K., BenBarak, M.J., Franco, C.B., Kalesnikoff, J., Yu, M., Tsai, M., Piliponsky, A.M., and Galli, S.J. (2011). Reduced mast cell and basophil numbers and function in Cpa3-Cre; Mcl-1fl/fl mice. Blood 118, 6930-6938.

Mackay, L.K., Minnich, M., Kragten, N.A., Liao, Y., Nota, B., Seillet, C., Zaid, A., Man, K., Preston, S., Freestone, D., et al. (2016). Hobit and Blimp1 instruct a universal transcriptional program of tissue residency in lymphocytes. Science 352, 459-463.

Maizels, R.M. (2020). Regulation of immunity and allergy by helminth parasites. Allergy 75, 524534.

Marple, A., Wu, W., Shah, S., Zhao, Y., Du, P., Gause, W.C., and Yap, G.S. (2017). Cutting Edge: Helminth Coinfection Blocks Effector Differentiation of CD8 T Cells through Alternate Host Th2- and IL-10-Mediated Responses. Journal of immunology 198, 634-639. 
Martins, R., Carlos, A.R., Braza, F., Thompson, J.A., Bastos-Amador, P., Ramos, S., and Soares, M.P. (2019). Disease Tolerance as an Inherent Component of Immunity. Annual review of immunology $37,405-437$.

Matheis, F., Muller, P.A., Graves, C.L., Gabanyi, I., Kerner, Z.J., Costa-Borges, D., Ahrends, T., Rosenstiel, P., and Mucida, D. (2020). Adrenergic Signaling in Muscularis Macrophages Limits Infection-Induced Neuronal Loss. Cell 180, 64-78 e16.

Medzhitov, R., Schneider, D.S., and Soares, M.P. (2012). Disease tolerance as a defense strategy. Science 335, 936-941.

Mitroulis, I., Ruppova, K., Wang, B., Chen, L.S., Grzybek, M., Grinenko, T., Eugster, A., Troullinaki, M., Palladini, A., Kourtzelis, I., et al. (2018). Modulation of Myelopoiesis Progenitors Is an Integral Component of Trained Immunity. Cell 172, 147-161 e112.

Muller, P.A., Koscso, B., Rajani, G.M., Stevanovic, K., Berres, M.L., Hashimoto, D., Mortha, A., Leboeuf, M., Li, X.M., Mucida, D., et al. (2014). Crosstalk between muscularis macrophages and enteric neurons regulates gastrointestinal motility. Cell 158, 300-313.

O'Leary, C.E., Schneider, C., and Locksley, R.M. (2019). Tuft Cells-Systemically Dispersed Sensory Epithelia Integrating Immune and Neural Circuitry. Annual review of immunology 37, 47-72.

Ohman, L., and Simren, M. (2010). Pathogenesis of IBS: role of inflammation, immunity and neuroimmune interactions. Nature reviews Gastroenterology \& hepatology 7, 163-173.

Schneider, C., O'Leary, C.E., von Moltke, J., Liang, H.E., Ang, Q.Y., Turnbaugh, P.J., Radhakrishnan, S., Pellizzon, M., Ma, A., and Locksley, R.M. (2018). A Metabolite-Triggered Tuft Cell-ILC2 Circuit Drives Small Intestinal Remodeling. Cell 174, 271-284 e214.

Silveira, M.R., Nunes, K.P., Cara, D.C., Souza, D.G., Correa, A., Jr., Teixeira, M.M., and Negrao-Correa, D. (2002). Infection with Strongyloides venezuelensis induces transient airway eosinophilic inflammation, an increase in immunoglobulin E, and hyperresponsiveness in rats. Infection and immunity 70, 6263-6272.

Soares, M.P., Gozzelino, R., and Weis, S. (2014). Tissue damage control in disease tolerance. Trends in immunology 35, 483-494.

Tsolis, R.M., Townsend, S.M., Miao, E.A., Miller, S.I., Ficht, T.A., Adams, L.G., and Baumler, A.J. (1999). Identification of a putative Salmonella enterica serotype typhimurium host range factor with homology to IpaH and YopM by signature-tagged mutagenesis. Infection and immunity 67, 6385-6393.

Vivier, E., Artis, D., Colonna, M., Diefenbach, A., Di Santo, J.P., Eberl, G., Koyasu, S., Locksley, R.M., McKenzie, A.N.J., Mebius, R.E., et al. (2018). Innate Lymphoid Cells: 10 Years On. Cell 174, 1054-1066.

White, J.P., Xiong, S., Malvin, N.P., Khoury-Hanold, W., Heuckeroth, R.O., Stappenbeck, T.S., and Diamond, M.S. (2018). Intestinal Dysmotility Syndromes following Systemic Infection by Flaviviruses. Cell. 
Wong, T., Hildebrandt, M.A., Thrasher, S.M., Appleton, J.A., Ahima, R.S., and Wu, G.D. (2007). Divergent metabolic adaptations to intestinal parasitic nematode infection in mice susceptible or resistant to obesity. Gastroenterology 133, 1979-1988.

Wu, D., Molofsky, A.B., Liang, H.E., Ricardo-Gonzalez, R.R., Jouihan, H.A., Bando, J.K., Chawla, A., and Locksley, R.M. (2011). Eosinophils sustain adipose alternatively activated macrophages associated with glucose homeostasis. Science 332, 243-247. 


\section{Materials and Methods}

\section{Mice}

C57BL/6J (000664), CD45.1 B6 (B6.SJL-Ptprc ${ }^{\mathrm{a}} \mathrm{Pepc}^{\mathrm{b}} /$ BoyJ), LysM ${ }^{\text {Cre }}$ (B6.129P2-Lyz2 ${ }^{\mathrm{tm} 1(\text { cre)lfo/J, }}$

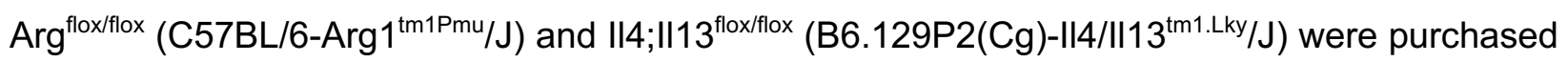
from The Jackson Laboratories and maintained in our facilities. Adrb2 $2^{\text {flox/flox }}\left(\right.$ Adrb2 $\left.{ }^{\text {tm } 1 \text { Kry }}\right)$ were

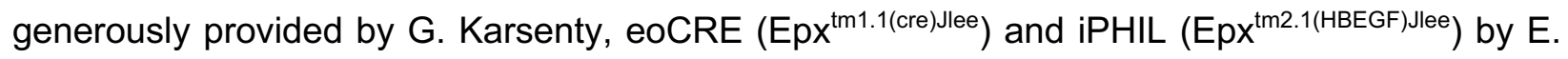
Jacobsen, Hobit-Blimp-1 DKO (Hobit ${ }^{-/}$Blimp ${ }^{\text {flox/flox }}$ LCKCre $\left.{ }^{\text {tg/+ }}\right)$ by K. van Gisbergen. cpa3 ${ }^{\text {DTR-tdTomato }}$ mice were generated as described below. Pet store mice were purchased at a local pet store (Petco). Mouse lines were interbred in our facilities to obtain the final strains described in the text. Genotyping was performed according to the protocols established for the respective strains by The Jackson Laboratories or personal communication with the donating investigators. Mice were maintained at the Rockefeller University animal facilities under specific pathogen-free conditions. Mice were fed a standard chow diet and used at 7-11 weeks of age for most experiments. Animal care and experimentation were consistent with $\mathrm{NIH}$ guidelines and were approved by the Institutional Animal Care and Use Committee at the Rockefeller University.

\section{Infections}

Salmonella enterica Typhimurium. For infections with Salmonella spiB, mice were pre-treated with a single dose of streptomycin (20 mg/mouse dissolved in $100 \mu$ of DPBS) administered by oral gavage $18-24 \mathrm{~h}$ prior to infection. Mice were then orally inoculated with $10^{9} \mathrm{CFU}$ of spiB. A single aliquot of spiB was grown in $3 \mathrm{ml}$ of $\mathrm{LB}$ overnight at $37^{\circ} \mathrm{C}$ with agitation. Bacteria were then subcultured $(1: 300)$ into $3 \mathrm{ml}$ of $\mathrm{LB}$ for $3.5 \mathrm{~h}$ at $37^{\circ} \mathrm{C}$ with agitation and diluted to final concentration in $1 \mathrm{ml}$ of DPBS.

Yersinia pseudotuberculosis. Y. pseudotuberculosis (strain IP32777) was grown as previously described (Fonseca et al., 2015). Briefly, a single aliquot of Yp was grown in $3 \mathrm{~mL}$ of 2xYT media 
overnight at $28^{\circ} \mathrm{C}$ with vigorous agitation and diluted $(1: 10)$ to final concentration in DPBS. Mice were fasted for $12-16 \mathrm{~h}$ prior to infection with $10^{8} \mathrm{CFU}$ by oral gavage.

Strongyloides venezuelensis. S. venezuelensis was maintained in our facility in NSG mice by subcutaneous infection with 1000 stage 3 (L3) larvae, resulting in chronic infection of this strain. For each experiment, feces of infected NSG mice were collected and spread on Whatman paper, which was places into a beaker with water and incubated at $28^{\circ} \mathrm{C}$ for $2-3$ days. Mice were infected subcutaneously with 700 L3 larvae in $200 \mu$ l water per mouse. S. venezuelensis was passaged periodically by infecting naïve adult NSG mice.

Heligmosomoides polygyrus. Mice were infected by oral gavage with 200 third-stage larvae of H. polygyurs in $100 \mu \mathrm{l}$ water.

\section{Generation of $c p a 3^{\text {DTR-tdTomato }}$ mice}

Cpa3 ${ }^{\text {DTR-tdTomato }}$ knock-in mice were generated using CRISPR/Cas9 technology directly in C57BL/6 mice as described before (REF). Briefly, two guide RNA (gRNA) targeting exon 11 in the 3' UTR of the cpa3 locus were designed using the online CRISPR Design Tool. These gRNAs were cloned into a plasmid (Addgene) containing Cas9n. The plasmid was then co-injected into the pronucleus with a repair template plasmid (i.e. targeting vector, Addgene) containing an IRESDTR-tdTomato fusion cassette. WT and knock-in genotypes were confirmed by PCR. DTR and IRES expression were confirmed using the repair template plasmid as a positive control. Insertion of the cassette into the correct locus was verified by using primers located inside the cassette and outside of the 3' and 5' homology regions, respectively.

\section{Antibodies}

Flow cytometry and whole-mount immunofluorescence antibodies. Antibodies against CD64-APC (Clone X54-5/7.1, cat. \# 13906), CD4-BV605 (Clone RM4-5, cat. \# 100548), CD150 (Clone TC15-12F12.2, cat. \# 115921), CD48-APC (Clone HM480-1, cat. \# 103411), Lineage 
Cocktail-PB (cat. \# 133305) were purchases from BioLegend. Antibodies against CD11b-APCeFluor780 (Clone M1/70, cat. \# 47-0112-82), FceR1-APC (Clone MAR1, cat. \# 17-5898-80), CD45-PE-Cy7 (Clone 30-F11, cat. \# 25-0451-82), CD45-AF700 (Clone 30-F11, cat. \# 56-045182), CD45.1-PE-Cy7 (Clone A20, cat. \# 25-0453-82), GATA-3-PE (Clone TWAJ, cat. \# 12-996642), FOXP3-eFluor450 (Clone FJK-16s, cat. \# 48-5773-82), MHC II-AF700 (Clone M5/114.15.2, cat. \# 56-5321-82), Ly-6G-eFluor450 (Clone RB6-8C5, cat. \# 48-5931-82), CD11c-AF488 (Clone N418, cat. \# 53-0114-82), IL-4-APC (Clone 11B11, cat. \# 17-7041-82), Arg1-PE-Cy7 (Clone A1exF5, cat. \# 25-36-97-82), Ly6A/E(Sca-1)-PE (Clone D7, cat. \# 12-5981-82), CD16/CD32FITC (clone 93, cat. \# 11-0161-82) were purchases from Thermo Fisher Scientific. Antibodies against CD11b-FITC (Clone M1/70, cat. \# 553310), Siglec-F-APC-Cy7 (Clone E50-2440, cat. \# 565527), -PE (Clone E50-2440, cat. \# 552126) and -BV421 (Clone E50-2440, cat. \# 56268), ckit-PE-Cy7 (Clone 2B8, cat. \# 558163), CD45.2-PerCP-Cy5.5 (Clone 104, cat. \# 552950), CD45.2-AF700 (Clone 104, cat. \# 560693), CD45R-FITC (Clone RA3-6B2, cat. \# 553088), CD8aAF488 (Clone 53-6.7, cat. \# 557668), I-A/I-E-FITC (Clone M5/114.15.2, cat. \# 553623), CD125AF488 (Clone T21, cat. \# 558533) were purchased from BD. Cell surface and intracellular antibodies were used at 1:200 and 1:100 dilution, respectively. Antibody against ANNA-1 was a gift from Dr. Vanda A. Lennon. Fluorophore-conjugated secondary antibody H\&L goat antihuman-AF568 (Thermo Fisher Scientific) was used to detect anti-ANNA-1 antibody.

Antibodies for in vivo experiments. Antibodies against CD4 (Clone GK1.5, cat. \# BE0003-1), CSF1R (Clone ASF98, cat. \# BE0213) and IL-5 (Clone TRFK5, cat. \# BE0198) and corresponding isotype controls were purchased from BioXCell. CD4 mAb was injected i.p. on indicated days at $200 \mu \mathrm{g} /$ mouse/timepoint. IL-5 mAb was injected i.p. on indicated days at $500 \mu \mathrm{g} / \mathrm{mouse} / \mathrm{timepoint}$. CSF1R mAb was injected i.p. on indicated days at $50 \mathrm{mg} / \mathrm{kg}$. Mice were previously habituated to i.p. injections for at least 5 days prior to the antibody treatments. 


\section{Ivermectin treatment}

Ivermectin (Milipore Sigma) was dissolved in DMSO at $100 \mathrm{mg} / \mathrm{ml} .14$ days after Sv infection, ivermectin was orally gavaged at $50 \mu \mathrm{g} /$ mouse. 14 days after $\mathrm{Hp}$ infection ivermectin was injected subcutaneously at $20 \mathrm{mg} / \mathrm{kg}$.

\section{Diphtheria toxin treatment}

DT (Sigma Aldrich) was reconstituted in DPBS. iPHIL mice were treated i.p. on indicated days at 15 ng/gram body weight. Cpa3-DTR mice were treated i.p. on indicated days at $300 \mu \mathrm{g} / \mathrm{mouse}$.

\section{Intestine dissection}

Mice were sacrificed and duodenum (1 cm moving distal from the gastroduodenal junction) or ileum (1 $\mathrm{cm}$ moving proximal from the ileocecal junction) was removed. For dissection of the muscularis, following the above procedures, the intestinal tissue was placed on a chilled aluminum block with the serosa facing up (Gabanyi et al., 2016). Curved forceps were then used to carefully remove the muscularis. Tissue was then used for whole-mount imaging or flow cytometry.

\section{Muscularis processing for flow cytometry}

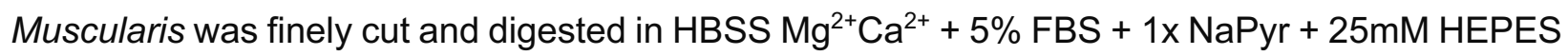
$+50 \mu \mathrm{g} / \mathrm{ml}$ DNasel (Roche) $+400 \mathrm{U} / \mathrm{ml}$ Collagenase D (Roche) + $2.5 \mathrm{U} / \mathrm{ml}$ Dispase (Corning) at $37^{\circ} \mathrm{C}$. The muscularis was digested for $40 \mathrm{~min}$. The tissue was then homogenized with an $18-$ gauge needle and filtered through a $70 \mu \mathrm{m}$ cell strainer and washed with $\mathrm{HBSS} \mathrm{Mg}^{2+} \mathrm{Ca}^{2+}$. The cells were incubated with Fc block and antibodies against the indicated cell surface markers in FACS buffer (PBS, 1\% BSA, 10 mM EDTA, 0.02\% sodium azide). 


\section{Bone marrow processing}

Bone marrow cells from femurs were passed through $70 \mu \mathrm{m}$ filter and treated with red blood cell lysis buffer, followed by staining with fluorescently labeled antibodies.

\section{Isolation of intraepithelial and lamina propria cells}

Intraepithelial and lamina propria cells were isolated as previously described (Bilate et al., 2016). Briefly, sections of small intestines were harvested and washed in PBS and 1mM dithiothreitol (DTT) followed by 30 mM EDTA. Intraepithelial cells were recovered from the supernatant of DTT and EDTA washes and mononuclear cells were isolated by gradient centrifugation using Percoll. Cells from lamina propria were obtained after collagenase 8 digestion of the tissue. Single-cell suspensions were then stained with fluorescently labeled antibodies for 30 min on ice.

\section{Intracelluar staining for flow cytometry}

Intranuclear staining for transcription factors was conducted using Foxp3 / Transcription Factor Staining Buffer Set according to manufacturer's instructions (eBioscience, USA). Intracellular staining for cytokines was conducted in Perm/Wash buffer after fixation and permeabilization in Fix/Perm buffer (BD Biosciences, USA) according to kit instructions. Flow cytometry data were acquired on an LSR-II flow cytometer (Becton Dickinson, USA) and analyzed using FlowJo software package (Tri-Star, USA).

\section{Gating strategies}

For flow cytometric analysis following gating strategy was used to identify macrophages: single, live, myeloid cells (based on FSC, SSC and live/dead fixable dye Aqua stain), CD45+, CD11b+ and CD64+. Eosinophils: single, live, myeloid cells, CD45+, CD11b+ and Siglec-F+. Mast cells: single, live, myeloid cells, CD45+, CD117+ and FceR1+. For all cell types, B220+ and CD8a+ cells were excluded. 


\section{Eosinophil sorting}

Following lamina propria cell isolation, cells were processed as previously described (Geslewitz et al., 2018). Cells were then sorted using a FACSAria ${ }^{\text {TM }}$ cell sorter flow cytometer (Becton Dickinson) and subsequently transferred by retro-orbital injection at numbers indicated in Figure 5.

\section{Cytokine quantification}

After centrifugation of peripheral blood, the serum samples were immediately aliquoted and stored at $4{ }^{\circ} \mathrm{C}$ until the experiment was conducted. The concentrations of IL-4 and IL-13 were measured once after collection of each individual samples by using the cytometric bead array assay (BD Biosciences) according to the manufacturer's instructions.

\section{CFU counting}

Fecal pellets from spiB- or Yp-infected mice were weighed and then disrupted in $400 \mathrm{~mL}$ of DPBS. Serial dilutions were made from the original suspension and then $5 \mathrm{ml}$ of each dilution was plated onto Salmonella-Shigella or 2xYT-Irgasan plates, respectively. The plates were then incubated overnight, and the number of black colonies were counted for the serial dilution with the clearest delineation of single units. This number was then multiplied by the dilution factor and by 80 to give the number of colony-forming units (CFU) in the original suspension. CFU numbers were then divided by the original fecal pellet weight to give the number of CFU per mg of feces.

\section{Quantification of Sv eggs in feces}

Fecal pellets from Sv-infected mice were weighed and then disrupted in $400 \mu$ of DPBS. $100 \mu \mathrm{l}$ of iodine solution was added to increase the visibility of fecal eggs. Eggs in small portions of each 
sample were counted under a microscope, and the number of eggs per gram of feces was determined for each sample.

\section{Intestine motility measurements}

For measurement of total intestinal transit time, mice were given an oral gavage of $6 \%$ carmine red (Sigma-Aldrich) dissolved in $0.5 \%$ methylcellulose (prepared in sterile $0.9 \% \mathrm{NaCl}$ ). Total intestinal transit time was measured as the time from oral gavage it took for mice to pass a fecal pellet that contained carmine.

\section{Neuronal imaging and counting quantification}

After dissection muscularis was pinned down on a plate coated with Sylgard and then fixed overnight with $4 \%$ PFA. After washing in DPBS whole mount samples were then permeabilized first in $0.5 \%$ Triton $\mathrm{X}-100$ (perm buffer) for $2 \mathrm{~h}$ at room temperature (RT) with gentle shaking. Samples were then blocked for $2 \mathrm{~h}$ in blocking buffer ( $5 \%$ bovine serum albumin and $5 \%$ goat serum in perm buffer) for $2 \mathrm{~h}$ at RT with gentle agitation. Anti-ANNA-1 antibody was added to the blocking buffer at appropriate concentrations and incubated for 2 days at $4^{\circ} \mathrm{C}$. After primary incubation the tissue was washed 4 times in perm buffer and then incubated in blocking buffer with secondary antibody for 2-3 h at RT. Samples were again washed 4 times in perm buffer and then mounted with FluoroMount $\mathrm{G}$ on slides with coverslips. Slides were kept in the dark at $4{ }^{\circ} \mathrm{C}$ until they were imaged. A minimum of 5-10 images were randomly acquired across a piece of whole-mount muscularis. These images were then opened in Fiji, and the cell counter was used to count the number of ANNA-1+ cells in a given field. This number was then multiplied by a factor of 2.95 (20x objective) or 3.125 (25x objective), to calculate the number of counted neurons per square millimeter $\left(\mathrm{mm}^{2}\right)$. The average of 8-10 images was then calculated and plotted. Wholemount intestine samples were imaged on an inverted LSM 880 NLO laser scanning confocal and 
multiphoton microscope (Zeiss) and on an inverted TCS SP8 laser scanning confocal microscope (Leica).

\section{CG-SMG dissections and cFos staining}

Mice were cervically dislocated and a midline incision was made; the viscera were removed out of the peritoneal cavity. The superior mesenteric artery was located by identifying the intersection of the descending aorta and left renal artery. Fine forceps and microdissection scissors were used to remove the CG-SMG which is wrapped around the superior mesenteric artery and associated lymphatic vessels. CG-SMG samples were washed in cold $1 \mathrm{x}$ PBS and fixed overnight at $4{ }^{\circ} \mathrm{C}$ (rotating) in fresh 4\% PFA. CG-SMG samples were then washed four times in 1x PBS at room temperature and blocked overnight in 5\% Normal Donkey Serum with 0.5\% Triton X-100/0.05\% Tween-20/4 $\mathrm{Mg}$ heparin $(\mathrm{PTxwH})$ at $4{ }^{\circ} \mathrm{C}$. Samples then incubated with anti-cFos (1:500; Cell Signaling Technologies, 2250S) and anti-TH (1:200; Milipore; AB1542) for 48 hours at $4{ }^{\circ} \mathrm{C}$ (rotating). Samples were washed four times in $\mathrm{PTxwH}$ at room temperature and incubated with highly cross-adsorbed donkey anti-rabbit AF647 (Invitrogen A31573) and donkey anti-sheep AF568 (Invitrogen; A21099) antibodies at $4{ }^{\circ} \mathrm{C}$ for 48 hours (rotating). Samples were washed four times in PTxwH at room temperature and mounted in Fluoromount G. TH staining was used to identify the sympathetic neurons and number of cFos+ nuclei was counted in multiple z-stack images with confocal microscope. ImageJ Cell Counter plugin was used to count the cFos+ nuclei and data were not normalized to area or volume. Each data point represents the total number of cFos+ cells per CG-SMG.

\section{Intestine neuronal quantification: neurons per ganglion.}

A myenteric ganglion was defined as a continuous group of ANNA1+ cells that are separated by less than $15 \mu \mathrm{m}$ in distance. Only complete ganglia were counted per field of view. Thus, the 
following ganglia were excluded: 1. Ganglia that were truncated; 2 . No clear separation (>15 $\mu \mathrm{m})$ was noted between the last ANNA1+ cell and the edge of the field of view. In the case of single ANNA $1+$ cells that are separated by $15 \mu \mathrm{m}$ on all sides, this was counted as extraganglionic. The number of quantifiable ganglia was averaged across a minimum of 10 images per gut segment per animal.

\section{Statistical analysis}

Significance levels indicated are as follows: ${ }^{*} p<0.05,{ }^{* *} p<0.01,{ }^{* *} p<0.001,{ }^{* * *} p<0.0001$. All data are presented as mean \pm SD or mean \pm SEM. At least two independent experiments were performed throughout in this study. All statistical tests used were two- tailed. Multivariate data were analyzed by one-way ANOVA and Tukey's multiple comparisons post hoc test. Comparisons between two conditions were analyzed by unpaired Student's t test. GraphPad PRISM version 9 was used for generation of graphs and statistics. 